\title{
Analysis of plasma instabilities and verification of the BOUT code for the Large Plasma Device
}

\author{
P. Popovich, ${ }^{1}$ M.V. Umansky, ${ }^{2}$ T.A. Carter, ${ }^{1}$, a) and B. Friedman ${ }^{1}$ \\ 1) Department of Physics and Astronomy and Center for Multiscale Plasma Dynamics, \\ University of California, Los Angeles, CA 90095-1547 \\ ${ }^{2)}$ Lawrence Livermore National Laboratory, Livermore, CA 94550, \\ $U S A$
}

(Dated: 8 August 2018)

The properties of linear instabilities in the Large Plasma Device [W. Gekelman et al., Rev. Sci. Inst., 62, 2875 (1991)] are studied both through analytic calculations and solving numerically a system of linearized collisional plasma fluid equations using the 3D fluid code BOUT [M. Umansky et al., Contrib. Plasma Phys. 180, 887 (2009)], which has been successfully modified to treat cylindrical geometry. Instability drive from plasma pressure gradients and flows is considered, focusing on resistive drift waves, the Kelvin-Helmholtz and rotational interchange instabilities. A general linear dispersion relation for partially ionized collisional plasmas including these modes is derived and analyzed. For LAPD relevant profiles including strongly driven flows it is found that all three modes can have comparable growth rates and frequencies. Detailed comparison with solutions of the analytic dispersion relation demonstrates that BOUT accurately reproduces all characteristics of linear modes in this system.

PACS numbers: 52.30.Ex, 52.35.Fp, 52.35.Kt, 52.35.Lv, 52.65.Kj

a) Electronic mail: tcarter@physics.ucla.edu 


\section{INTRODUCTION}

Understanding complex nonlinear phenomena in magnetized plasmas increasingly relies on the use of numerical simulation as an enabling tool. The development of a robust predictive capability requires numerical models which are verified through comparison with analytic calculation and validated through comparison with experiment ${ }^{\underline{1}}$. A tractable analytic problem useful for verification of numerical models of plasma turbulence and transport is linear stability $\underline{2}^{-4}$. Understanding of linear instabilities in a set of model equations forms a framework for developing physical insights and mathematical apparatus that can be further used for attacking a more difficult nonlinear problem.

This paper presents a study of linear gradient-driven instabilities in a cylindrical magnetized plasma using the Braginskii two-fluid model. This work was undertaken with two motivations: (1) to gain understanding of the character of linear instabilities in the Large Plasma Device (LAPD) at UCLA $\underline{5}$ and (2) to verify linear calculations using the BOUT 3D Braginskii fluid turbulence code $\mathrm{e}^{\underline{6}}$ in cylindrical geometry. The BOUT code was originally developed in the late 1990s for modeling tokamak edge plasmas; the version of the code used in this study is described in detail by Umansky ${ }^{7}$.

Instability drive in LAPD comes from plasma pressure gradients ${ }^{8}$ and strong azimuthal flow which can be externally driven through biasing 9.10 . These free energy sources can drive resistive drift waves $\underline{11}$ and Kelvin-Helmholtz and rotational interchange instabilities ${ }^{12}$. The Kelvin-Helmholtz instability and unstable drift-Alfvén waves have been experimentally observed in LAPD $\underline{9,10,13,14}$. The analytic calculations and verification runs on BOUT are performed using LAPD-like profiles of plasma density, temperature and plasma potential (and therefore cross-field $E \times B$ flow). It is found that all three modes (drift waves, KelvinHelmholtz, rotational interchange) can be important in LAPD plasmas. Detailed comparison with solutions of the analytic dispersion relation demonstrates that BOUT accurately reproduces all characteristics of linear modes in this system. This work forms the foundation for nonlinear modeling of turbulence and transport in LAPD, initial results of which will be presented in a companion paper $\underline{\underline{15}}$.

This paper is organized as follows. Section Пintroduces the LAPD geometry and presents the fluid model used for calculations of linear instabilities. Section [II] discusses the implementation of these equations in the BOUT code, including a discussion of techniques used 
to extract characteristics of linear instabilities. Comparison of BOUT calculations to analytic linear eigenmode solutions are presented in Section IV] for three instabilities: resistive drift waves, Kelvin-Helmholtz and rotational interchange modes. Section $\nabla$ discusses the linear stability of experimentally measured LAPD profiles against these three instabilities and a discussion of the similarity to experimental observations. The effect of ion-neutral collisions on the linear solutions is discussed in Section VI. A summary of the paper is presented in Section VII. Appendices are provided which cover: a derivation of the specific set of fluid equations used in this work (Appendix A); a derivation of the vorticity equation used in BOUT (Appendix $(\mathrm{B})$; and a list of parameters and boundary conditions used in the verification study (Appendix C).

\section{GEOMETRY AND PHYSICS MODEL}

The geometry used in this study is that of the LAPD: a $\sim 17 \mathrm{~m}$ long cylindrical magnetized plasma with typical plasma radius (half-width at half-maximum) of $a \sim 30 \mathrm{~cm}$ (vacuum chamber radius $r=50 \mathrm{~cm}$ ). Typical plasma parameters in LAPD for a $1 \mathrm{kG}$ magnetic field are shown in Table 【.

The configuration is modeled as a cylindrical annulus to avoid the singularity of cylindrical coordinates near the axis in the BOUT numerical implementation (Fig 1). Using the scheme shown in Fig 1, LAPD geometry can be completely described within BOUT framework without major modification of the core code. The only change related to geometry in the code that is necessary is the implementation of the full cylindrical Laplacian operator to extend the simulation domain closer to the magnetic axis.

The magnetic field is taken uniform, directed along the cylinder axis. The axial boundary conditions are taken periodic for simplicity. A more realistic model should include the endplate sheath boundary conditions, supporting potentially important wall-driven instabilities; this will be the subject of future work. Radial boundary conditions used here are either zero value or zero radial gradient.

A Braginskii two-fluid mode ${ }^{16}$ is used in the analytic and BOUT calculations for instabilities in LAPD. As evident from Table I, collisions are important in LAPD plasmas: the electron collision mean free path is much smaller than the system size parallel to the magnetic field, $\lambda_{e i} \ll L_{\|}$. Therefore for long parallel wavelength, low frequency modes $\left(\omega \ll \Omega_{i}\right)$ 


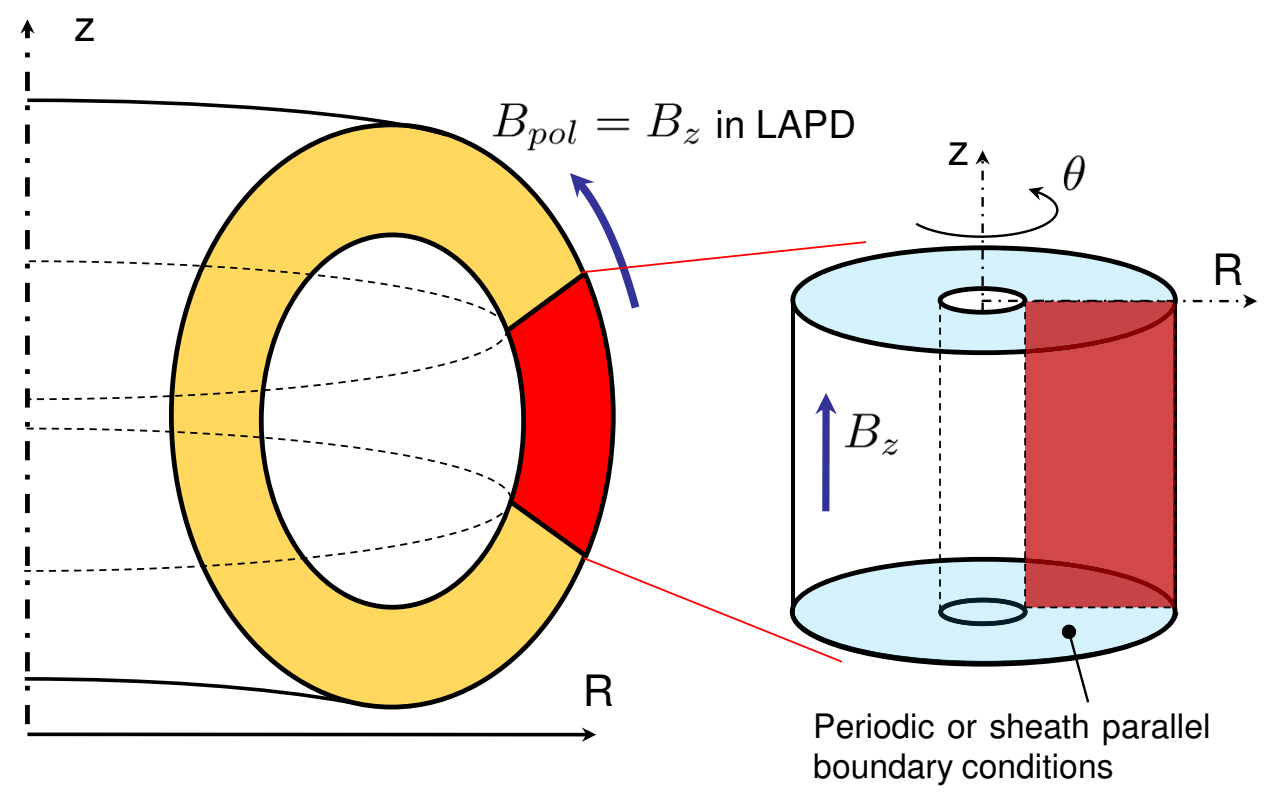

FIG. 1. (Color Online) Schematic view of LAPD geometry representation in the BOUT code. The poloidal direction of the tokamak geometry becomes the axial direction $z$ in LAPD, and the toroidal coordinate of a tokamak corresponds to the azimuthal angle $\theta$ in LAPD.

\begin{tabular}{|l|c||c|c|}
\hline Species & ${ }^{4} \mathrm{He}$ & $f_{c i}$ & $380 \mathrm{kHz}$ \\
$Z$ & 1 & $\rho_{i}$ & $0.2 \mathrm{~cm}$ \\
$n$ & $2.5 \times 10^{12} \mathrm{~cm}^{-3}$ & $\rho_{s}$ & $0.5 \mathrm{~cm}$ \\
$T_{\mathrm{e}}$ & $5 \mathrm{eV}$ & $\nu_{e i}$ & $7.4 \times 10^{6} 1 / \mathrm{s}$ \\
$T_{\mathrm{i}}$ & $\lesssim 1 \mathrm{eV}$ & $\nu_{i i}$ & $5 \times 10^{5} 1 / \mathrm{s}$ \\
$B_{0}$ & $0.1 \mathrm{~T}$ & $\nu_{i n}$ & $1.2 \times 10^{3} 1 / \mathrm{s}$ \\
$L_{\|}$ & $17 \mathrm{~m}$ & $\lambda_{e i}$ & $13 \mathrm{~cm}$ \\
$a$ & $\sim 0.3 \mathrm{~m}$ & $\omega_{*}$ & $\sim 4 \times 10^{4} \mathrm{rad} / \mathrm{s}$ \\
\hline
\end{tabular}

TABLE I. Typical LAPD parameters

considered here, it could be argued that the use of a collisional fluid theory is justified. However, it should be noted that the quantity most important for evaluating the importance of kinetic effects is the ratio of the parallel wave phase speed to the thermal speed of the particles, and for drift-type modes and Alfvén waves in LAPD this can be near unity for the 
electrons ${ }^{8,17}$. Strong collisions can disrupt velocity-space resonant processes and it might be expected that a fluid description becomes accurate even for $v_{\phi} \sim v_{\text {th }}$ as $k_{\|} \lambda_{\text {ei }} \rightarrow 0$, as has been shown for ion acoustic waves through Fokker-Planck calculations ${ }^{18}$. The present work is part of an ongoing effort to evaluate the validity of a fluid model (in particular that implemented in BOUT) in describing turbulence in LAPD. A goal of this study is to determine whether (and how) fluid simulations can fail to describe plasma behavior, and kinetic effects are likely to delineate when failure occurs.

The fluid equations used here represent conservation of density, electron and ion momentum and charge:

$$
\begin{aligned}
\left(\partial_{t}+\mathbf{v}_{e} \cdot \nabla\right) n & =0 \\
n m_{e}\left(\partial_{t}+\mathbf{v}_{e} \cdot \nabla\right) \mathbf{v}_{e} & =-\nabla p_{e}-n e\left(\mathbf{E}+\frac{1}{c} \mathbf{v}_{e} \times \mathbf{B}\right) \\
n m_{i}\left(\partial_{t}+\mathbf{v}_{i} \cdot \nabla\right) \mathbf{v}_{i} & =n e\left(\mathbf{E}+\frac{1}{c} \mathbf{v}_{i} \times \mathbf{B}\right)-n m_{i} \nu_{i n} \mathbf{v}_{i} \\
\nabla \cdot \mathbf{J} & \left.=0, \quad \mathbf{J}=e n\left(\mathbf{v}_{i \|}-\mathbf{v}_{i}\right)-n m_{e} \nu_{e n} \mathbf{v}_{e}\right)+e n\left(\mathbf{v}_{i \perp}-\mathbf{v}_{e \perp}\right)
\end{aligned}
$$

where $p_{e}=n k_{\mathrm{B}} T_{\mathrm{e}}$. A friction term due to ion-neutral collisions (elastic and charge-exchange) is included in the ion momentum equation. All terms involving finite ion temperature effects are neglected. The friction forces in the electron momentum equation are due to electron-ion $\left(\nu_{e i}\right)$ and electron-neutral collisions $\left(\nu_{e n}\right)$. However, as Coulomb collisions are dominant for the electrons $\left(\nu_{e i} \gg \nu_{e n}\right)$, electron-neutral collisions are ignored.

The following simplifying assumptions are made, which are relevant for LAPD plasma parameters: constant magnetic field $\mathbf{B}=B_{0} \mathbf{z}, v_{\| \mathrm{e}} \gg v_{\| \mathrm{i}}, T_{e} \gg T_{i}$, and no background parallel flows. In addition, it is assumed that the instabilities do not generate perturbations in the electron temperature. Throughout the paper plasma density, temperature and magnetic field are normalized to reference values $n_{x}, T_{e x}$ (chosen as the maximum of the corresponding equilibrium profiles), and $B_{0}$, the axial magnetic field. Frequencies and time derivatives are normalized to $\Omega_{i x}=e B_{0} / m_{i} c$ : $\hat{\partial}_{t}=\partial_{t} / \Omega_{i x}, \hat{\omega}=\omega / \Omega_{i x}$; velocities are normalized to the ion sound speed $C_{s x}=\sqrt{T_{e x} / m_{i}}$; lengths - to the ion sound gyroradius $\rho_{s x}=C_{s x} / \Omega_{i x}$; electrostatic potential to the reference electron temperature: $\hat{\phi}=e \phi / T_{e x}$. Further the " ^" symbol for dimensionless quantities will be dropped for brevity of notation. 
Combining Eqs.(1-4) and linearizing (see Appendix [A]), one obtains:

$$
\begin{aligned}
& \partial_{t} N+\mathbf{b}_{0} \times \nabla_{\perp} \phi_{0} \cdot \nabla N=-\mathbf{b}_{\mathbf{0}} \times \nabla_{\perp} \phi \cdot \nabla N_{0}-N_{0} \nabla_{\|} v_{\| \mathrm{e}} \\
& \partial_{t} v_{\| \mathrm{e}}+\mathbf{b}_{\mathbf{0}} \times \nabla_{\perp} \phi_{0} \cdot \nabla v_{\| \mathrm{e}}=-\mu \frac{T_{e 0}}{N_{0}} \nabla_{\|} N+\mu \nabla_{\|} \phi-\nu_{e} v_{\| \mathrm{e}} \\
& N_{0} \nabla_{\|} v_{\| \mathrm{e}}=-\nabla_{\perp} \cdot\left(N_{0} \partial_{t} \nabla_{\perp} \phi+\partial_{t} N \nabla_{\perp} \phi_{0}\right. \\
&+\mathbf{b}_{0} \times \nabla_{\perp} \phi_{0} \cdot \nabla\left(N_{0} \nabla_{\perp} \phi_{0}\right) \\
&+\mathbf{b}_{\mathbf{0}} \times \nabla_{\perp} \phi_{0} \cdot \nabla\left(N_{0} \nabla_{\perp} \phi\right) \\
&+\mathbf{b}_{\mathbf{0}} \times \nabla_{\perp} \phi \cdot \nabla\left(N_{0} \nabla_{\perp} \phi_{0}\right) \\
&+\mathbf{b}_{\mathbf{0}} \times \nabla_{\perp} \phi_{0} \cdot \nabla\left(N \nabla_{\perp} \phi_{0}\right) \\
&\left.+N_{0} \nu_{i n} \nabla_{\perp} \phi_{0}+N_{0} \nu_{i n} \nabla_{\perp} \phi+N \nu_{i n} \nabla_{\perp} \phi_{0}\right),
\end{aligned}
$$

where $N_{0}, \phi_{0}, T_{e 0}$ are zero-order (equilibrium) quantities and $N, \phi, v_{\| \mathrm{e}}$, are first order perturbations; $\mu=m_{i} / m_{e}$.

Note that Eqs. (5) contain a zero-order term, $\nabla_{\perp} \cdot\left(\nu_{i n} N_{0} \nabla_{\perp} \phi_{0}\right)$, which restricts the choice of background profiles in the presence of neutrals. If this term is not zero for a particular choice of $N_{0}(r)$ and $\phi_{0}(r)$ functions, then the plasma is not in mechanical equilibrium. In such a case, an extra zero-order force, e.g., from externally applied radial electric field, should be added to the momentum equation to balance the force of friction with the neutrals that slows down the plasma rotation.

Next, Eqs. (5) are projected onto cylindrical coordinate system $(r, \theta, z)$. Solutions of the form $f(\mathbf{x})=f(r) \exp \left(i m_{\theta} \theta+i k_{\|} z-i \omega t\right)$ are sought, where $k_{\|}=2 \pi n_{z} / L_{\|}, n_{z}$ is the parallel mode number. Denoting $f^{\prime}=\partial_{r} f$ and introducing the Doppler-shifted frequency $\tilde{\omega}=\omega-\frac{m_{\theta}}{r} \phi_{0}^{\prime}$, the 1D equation for radial eigenfunctions of the perturbed potential $\phi(r)$ can be written:

$$
C_{2}(r) \phi^{\prime \prime}+C_{1}(r) \phi^{\prime}+C_{0}(r) \phi=0
$$

where the coefficients $C_{i}(r)$ are functions of equilibrium quantities and of $\tilde{\omega}$ (full expressions for $C_{i}$ are presented in Appendix $\underline{\mathrm{A}}$ ).

Equation (6) is a 2nd order ordinary differential equation (ODE) in $r$. Supplemented with proper boundary conditions on the radial boundaries it forms a well-posed eigenvalue problem. In general Eq. (6) has to be solved numerically, due to the complex form of the coefficients $C_{i}$. Note that although Eq. (6) is better suited for theoretical analysis than the original system, Eqs. (5) , a complication for practical numerical solution of Eq. (6) is that 
the eigenvalue $\omega$ enters nonlinearly the coefficients $C_{i}$. Therefore, a numerical solution for the eigenvalues is easier to carry out using the original system Eqs. (5), which can be cast to a standard linear algebra eigenvalue problem amenable to solution by a standard eigenvalue package.

\section{SOLVING BY TIME-EVOLUTION WITH BOUT}

The present version of the BOUT $\operatorname{code}^{7}$ is a rather general framework suitable for integration of a system of time-evolution PDEs in $3 \mathrm{D}$ space of the form $\partial_{t} \mathbf{f}=\mathbf{F}(\mathbf{f}, \mathbf{x})$, where the right-hand-side $\mathbf{F}$ contains a combination of spatial differential operators applied to the state vector $\mathbf{f}$. The right-hand-side $\mathbf{F}$ is discretized on a spatial mesh by finite-differencing, which results in a system of ODEs that are integrated in time by an implicit ODE solver package PVODE ${ }^{19}$.

For the calculations presented here, the following set of equations are used in BOUT which are equivalent to Eqs. (144):

$$
\begin{aligned}
\partial_{t} N & =-\mathbf{v}_{\boldsymbol{E}} \cdot \nabla N-\nabla_{\|}\left(v_{\| \mathrm{e}} N\right) \\
\partial_{t} v_{\| \mathrm{e}} & =-\mathbf{v}_{\boldsymbol{E}} \cdot \nabla v_{\| \mathrm{e}}-\mu \frac{T_{e 0}}{N_{0}} \nabla_{\|} N+\mu \nabla_{\|} \phi-\nu_{e} v_{\| \mathrm{e}} \\
\partial_{t} \varpi & =-\mathbf{v}_{\boldsymbol{E}} \cdot \nabla \varpi-\nabla_{\|}\left(N v_{\| \mathrm{e}}\right)+\mathbf{b} \times \nabla N \cdot \nabla v_{E}^{2} / 2-\nu_{i n} \varpi
\end{aligned}
$$

where the potential vorticity

$$
\varpi \stackrel{\text { def }}{=} \nabla_{\perp} \cdot\left(N \nabla_{\perp} \phi\right)
$$

is introduced. While the variables $N, v_{\| \mathrm{e}}$ and $\varpi$ are advanced in time, Eq. (10) is inverted on each evaluation of the right-hand side of Eqs. (17.9) to reconstruct the perturbed potential $\phi$ from $\varpi$.

The vorticity evolution equation, Eq. (9), replaces the current continuity equation (4) in BOUT. Derivation of this form of the vorticity equation from Eq. (44) is presented in Appendix B. Note that Eq. (9) is equivalent to expression (76) in Simakov and Catto 20 , apart from the ion-neutral collision term which is included in this work and all the terms involving ion temperature which are neglected in the present work. The third term in the right-hand side of Eq. (9) is important even in the linear regime if strong background flows are present. Thus, it is essential in both linear and nonlinear simulations of LAPD 
experiments, which typically have strong azimuthal flows on the order of Mach number M $\sim 0.2$ for spontaneous flows and $\mathrm{M} \sim 1$ in bias-induced rotation experiments 10 .

To compare BOUT solution of the initial-value problem with the direct solution of eigenvalue problem corresponding to the discretized dispersion relation (5), the equations are linearized (Eqs. 7f-10) and advanced in time using BOUT from a small initial seed perturbation. BOUT produces perturbations, in this case, of density and vorticity/potential, as functions of 3D space and time.

A specific azimuthal mode number $m_{\theta}$ is selected by Fourier filtering in the azimuthal angle during the BOUT simulation. The parallel wave number $k_{\|}$is set by the length of the device and the periodic boundary conditions in the parallel direction.

The radial form of the numerical solution is dominated by the fastest growing radial eigenmode. Once the solution "locks in" to the fastest mode, we calculate the growth rate by fitting the time evolution of the volume-averaged amplitude of potential fluctuations to an

exponential. The frequency of the mode is then calculated by fitting the perturbed potential (with the exponential growth factored out) with a sine wave at each spatial position.

\section{VERIFICATION OF BOUT AGAINST EIGENVALUE SOLUTION}

\section{A. Electrostatic resistive drift wave}

In the absence of strong flows, the resistive drift mode is likely to be the primary instability in LAPD. In this section, the BOUT solution in LAPD geometry is verified using a reduced subset of fluid equations Eqs. (7+10) which support only the resistive drift instability branch.

The simplest model of the resistive drift wave can be written as a subset of the system Eqs. (77, $)$ :

$$
\begin{aligned}
\partial_{t} N & =-\mathbf{v}_{E} \cdot \nabla N_{0} \\
\partial_{t} v_{\| \mathrm{e}} & =-\mu \frac{T_{e 0}}{N_{0}} \nabla_{\|} N+\mu \nabla_{\|} \phi-\nu_{e} v_{\| \mathrm{e}} \\
\partial_{t} \varpi & =-\nabla_{\|}\left(N v_{\| \mathrm{e}}\right)
\end{aligned}
$$

These equations can be combined together to form a well-known local dispersion relation ${ }^{21}$ that assumes 1D dependence of the background density with constant gradient length $L_{n}=$ 
$N_{0}(x) / N_{0}^{\prime}(x):$

$$
\left(\frac{\omega}{\omega_{*}}-1\right) i \frac{\sigma_{\|}}{\omega_{*}}+\left(\frac{\omega}{\omega_{*}}\right)^{2}=0,
$$

where $\omega_{*}=\frac{k_{\perp}}{L_{n}} \frac{T_{e 0}}{m_{i} \Omega_{i}}, \sigma_{\|}=\frac{\Omega_{i} \Omega_{e}}{\nu_{e i}} \frac{k_{\|}^{2}}{k_{\perp}^{2}}$.

BOUT calculations were first verified on this simple local solution, Eq. (12), finding good agreement for a range of plasma parameters. Due to its simplicity, this solution provides useful insight into the behavior of the growth rates and frequencies. In a bounded plasma, the dispersion relation Eq. (12) together with a set of boundary conditions yields a set of discrete linearly unstable modes. Among these discrete modes, the fastest growing one is the mode that corresponds to dimensionless parameter $\sigma_{\|} / \omega_{*}$ closest to 1 .

The next, and more interesting, step is to compare BOUT calculations to the eigenvalue solution of the full non-local drift wave problem Eqs. (1-4). Here all terms in Eqs. (7-10) are retained. There is no background potential $\phi_{0}$ for these calculations which eliminates the Kelvin-Helmholtz and the rotation-driven interchange instabilities, and only allows for the drift wave solution. There is no simple analytic dispersion relation in this case. For comparison, we are using the direct numerical solution of the linear problem Eq. (5) obtained with an eigenvalue solver, as described in Section II. The results of this comparisons for cylindrical geometry with relevant to LAPD parameters and profiles are presented in Fig. (22). BOUT recovers the frequencies and growth rates for a range of magnetic field values $(B=$ $0.04,0.08$, and $0.12 \mathrm{~T}$ ). There is one-to-one correspondence between the eigenvalues found by the analytic solver and the BOUT solution. Typically, the discrepancy between the two methods is less than $2 \%$ for radial grids of 50 points, and the results converge with grid size. For comparison, frequencies and growth rates for longer wavelengths, $n_{z}=0.5$ (fundamental mode), are also shown (dashed lines).

As an example of numerical convergence, the relative error of the growth rate and frequency as a function of radial grid size $h=1 / N_{r}$ is shown in Fig. 3. The relative error is defined here as the difference between BOUT solution and the projected value at $h=0$, $\delta_{\gamma}=\left|\gamma-\gamma_{h=0}\right| / \gamma_{h=0}$, and analogously for the frequency $\omega$. The growth rate and frequency extracted from the initial-value simulation converge approximately quadratically in $h$. The difference between the BOUT solution at $h \rightarrow 0$ and the eigenvalue solver result is $0.43 \%$ for the frequency and $0.23 \%$ for the growth rate. This residual error is due to the limited numerical resolution in the azimuthal and parallel directions (both remain fixed at 16 

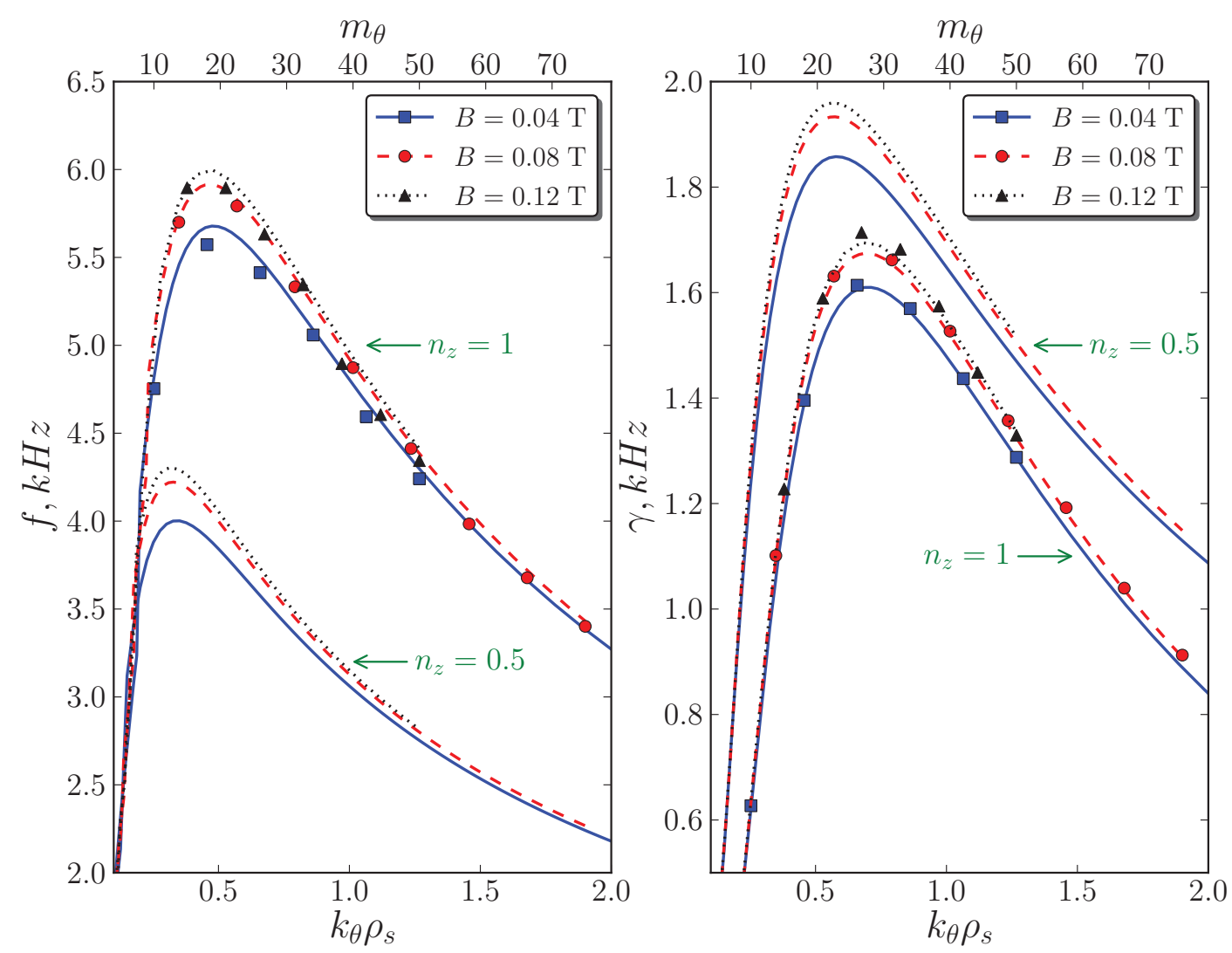

FIG. 2. (Color Online) Frequencies (left) and growth rates (right) of the resistive drift wave in LAPD configuration for experimental density profile, without equilibrium flows. Analytic results are shown with lines for $B=0.04 \mathrm{~T}$ (solid), $0.08 \mathrm{~T}$ (dash), $0.12 \mathrm{~T}$ (dotted); corresponding BOUT results are shown with squares, circles and triangles. The two groups of lines correspond to the axial harmonics $n_{z}=1$ and $n_{z}=0.5$. $m_{\theta}$ values on top axis are given for $B=0.04 \mathrm{~T}$.

grid points for this convergence study) and slight differences in the representation of the underlying equilibrium in BOUT and the eigenvalue solver.

\section{B. Kelvin-Helmholtz instability}

LAPD plasmas often involve large azimuthal flows, especially with biasing of the vacuum vessel wall 10,22 . The flows in the experiments with externally applied radial bias can reach Mach number of about 1 , or $v_{\theta} \sim 10^{6} \mathrm{~cm} / \mathrm{s}$. These speeds are much higher than the typical phase velocity of the drift wave, $v_{d} \sim 0.5 \times 10^{4} \mathrm{~cm} / \mathrm{s}$. Also, the growth rates of the 


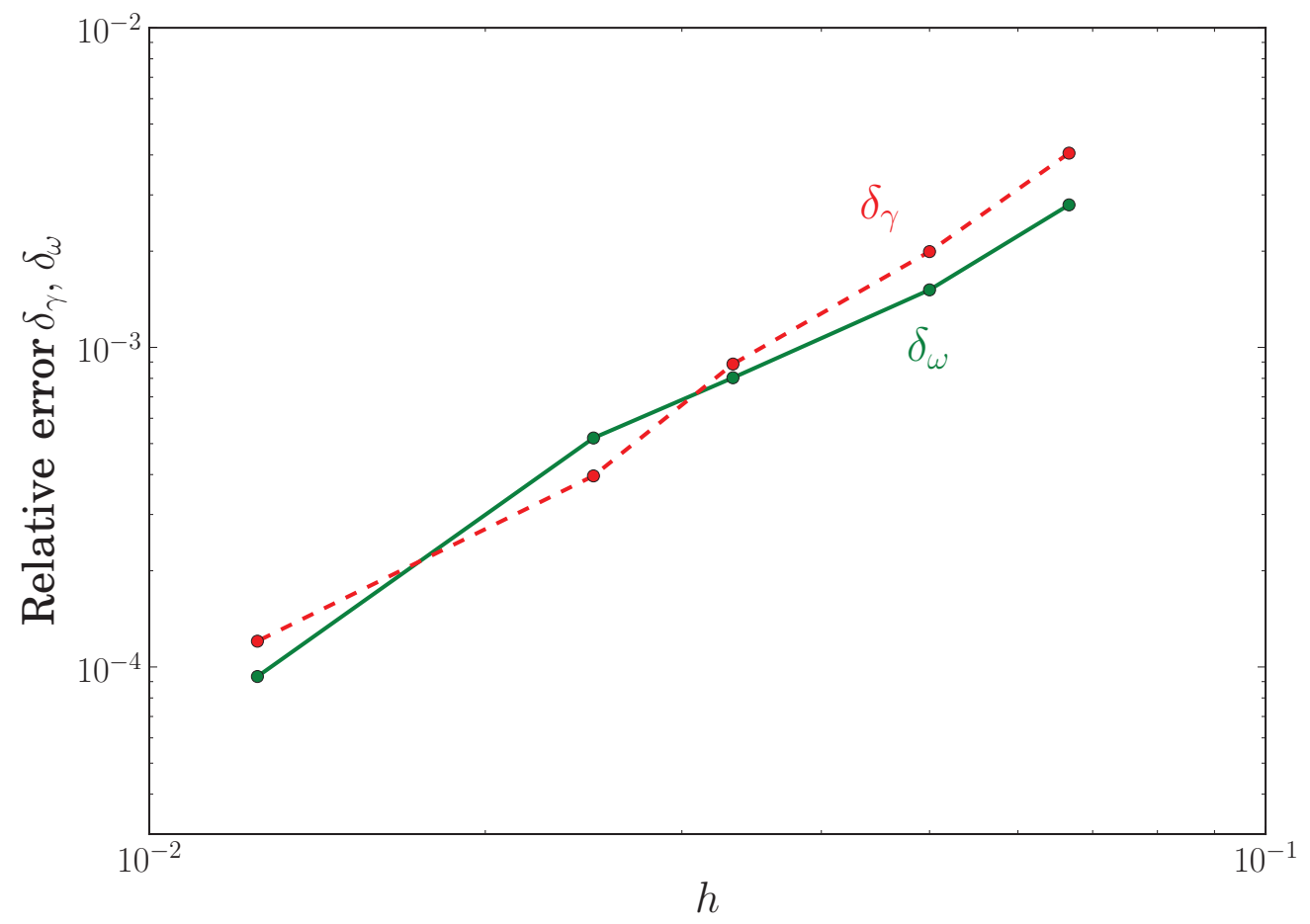

FIG. 3. (Color Online) Relative error of the growth rate and frequency determined from the initial-value simulation as a function of the radial grid size, indicating 2 nd order convergence.

instabilities generated by bias-induced flows can be comparable to that of the drift wave (see Section $\mathrm{V}$ ), therefore it is essential to include these flows in the model.

Kelvin-Helmholtz (KH) instability, driven by sheared flows, represents an interesting case for BOUT simulations in LAPD geometry, and provides a test of the implementation of the terms involving $\phi_{0}$ in BOUT. Observations of KH instability in LAPD plasmas have previously been reported by Horton et al. $\underline{\underline{9}}$.

A simple model for the $\mathrm{KH}$ instability can be obtained from the charge conservation equation, Eq. (44). Assuming no variation of equilibrium or perturbed quantities along the magnetic field (flute modes), only the polarization current contributes to this equation:

$$
\mathbf{J}_{\perp}=e n\left(\mathbf{v}_{i \perp}-\mathbf{v}_{e \perp}\right)=-\frac{c^{2} m_{i} n}{B^{2}} \frac{d \nabla_{\perp} \phi}{d t}=-\frac{c^{2} m_{i} n}{B^{2}}\left(\partial_{t}+\mathbf{v}_{\boldsymbol{E}} \cdot \nabla\right) \nabla_{\perp} \phi
$$

For simplicity, the case of constant plasma density and magnetic field is considered. The charge conservation equation can then be written as

$$
\left(\partial_{t}+\mathbf{v}_{\boldsymbol{E}} \cdot \nabla\right) \nabla_{\perp}^{2} \phi=0
$$


Linearizing Eq. (14) for slab geometry with periodic coordinate $y$ we obtain the eigenvalue equation $\underline{9} \underline{23}$ :

$$
\phi^{\prime \prime}(x)-\left(k_{y}^{2}+\frac{k_{y} \phi_{0}^{\prime \prime \prime}(x)}{k_{y} \phi_{0}^{\prime}-\omega}\right) \phi=0
$$

where the solution is assumed of the form $\phi(\mathbf{r}, t)=\phi(x) \exp \left(i k_{y} y-i \omega t\right)$

Analytic solution of this equation can be found for a specific choice of stream function $\phi_{0}$ by matching $\phi$ and its derivative jump at the points of singularity:

$$
\phi_{0}(x)=\left\{\begin{array}{lr}
0, & x \leq-1 \\
x^{2} / 2+x+1 / 2, & -1 \leq x \leq 0 \\
-x^{2} / 2+x+1 / 2, & 0 \leq x \leq 1 \\
1, & x>1
\end{array}\right.
$$

For direct comparison with BOUT, a solution must be found with boundary conditions imposed on a finite interval. We consider boundary conditions $\phi(-2)=\phi(2)=$ const. In this case, the eigenvalues are

$$
\omega=\left(e^{2 k_{y}}-1\right) /\left(2+2 e^{2 k_{y}}\right)
$$

for the neutrally stable branch, and

$$
\begin{aligned}
\omega= & \left(1-e^{4 k_{y}}+2 k_{y}+2 k_{y} e^{4 k_{y}} \pm \sqrt{G\left(k_{y}\right)}\right) /\left(4+4 e^{4 k_{y}}\right), \\
G\left(k_{y}\right)= & 9-16 e^{2 k_{y}}+14 e^{4 k_{y}}-16 e^{6 k_{y}}+9 e^{8 k_{y}}+12 k_{y}-12 k_{y} e^{8 k_{y}} \\
& +4 k_{y}^{2}+8 k_{y}^{2} e^{4 k_{y}}+4 k_{y}^{2} e^{8 k_{y}}
\end{aligned}
$$

for the stable/unstable branches. One of the branches is unstable for $0 \leq k_{y} \leq 1.815$, maximum growth rate is 0.2346 at $k_{y} \approx 1.241$. This result is similar to the calculation presented by Horton et al. for slightly different boundary conditions $\stackrel{9}{ }$.

This instability is found with BOUT by solving Eq. (14) written in terms of vorticity:

$$
\partial_{t} \varpi+\mathbf{v}_{\boldsymbol{E}} \cdot \nabla \varpi=0
$$

Eq. (18) is explicitly linearized in BOUT and solved in slab geometry with the same boundary conditions $\phi(-2)=\phi(2)=$ const. In BOUT, slab geometry is approximated as a small azimuthal segment of a large aspect ratio thin annulus. The exponential growth rate and the mode frequency is extracted from the time evolution of the perturbed potential $\phi$. Using 


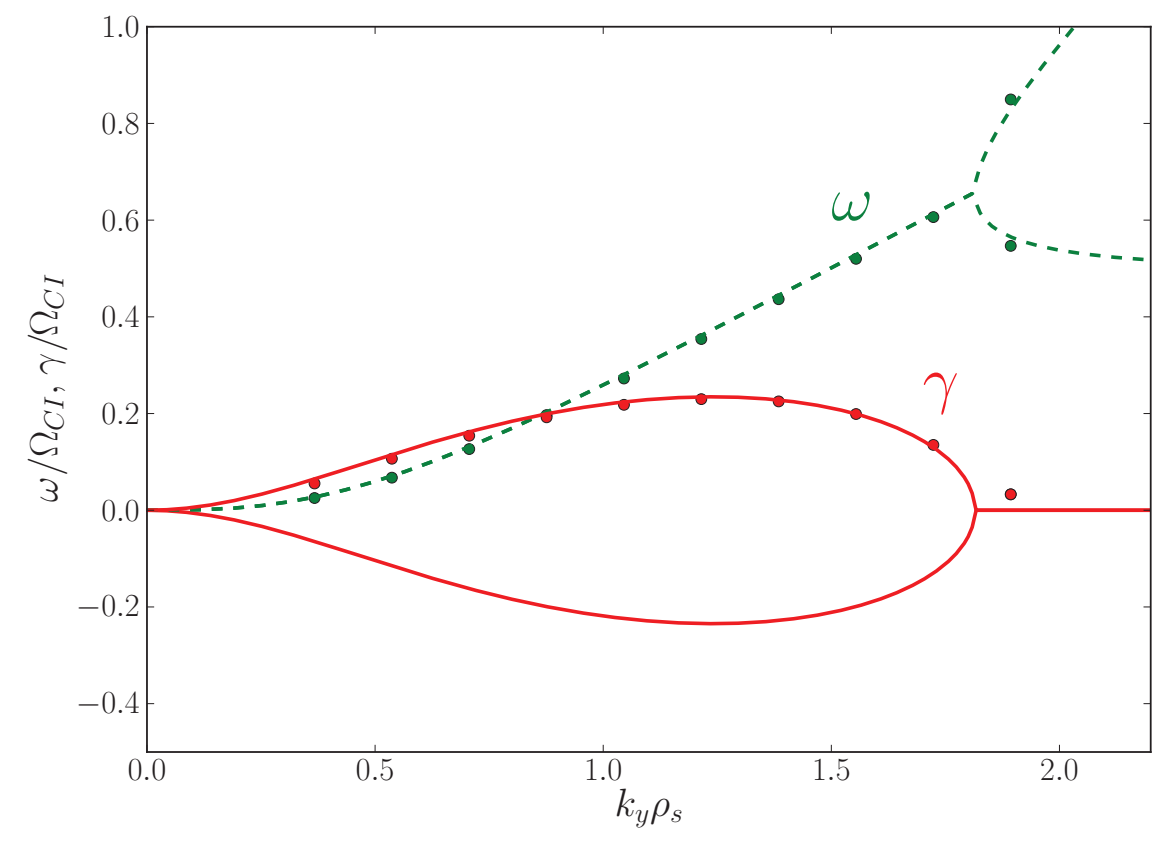

FIG. 4. (Color Online) Analytic solution (frequency - dashed line, growth rate - solid line) and BOUT simulations (circles) for the Kelvin-Helmholtz instability in slab geometry. $\phi_{0}$ profile for this case is given by Eq. (16).

this method, the frequencies and growth rates of the direct eigenvalue solution are recovered, as shown in Fig. 4 .

Note that the third derivative of $\phi_{0}$ that is present in Eq. (18) (as can be seen from Eq. (15) ) is singular, but it does not directly enter BOUT equations. BOUT uses $\phi_{0}$ profile as input, which is a smooth function. Therefore, the code has no difficulty reproducing the analytic solution even though it implies a singularity in the $\phi_{0}^{\prime \prime \prime}$ profile.

Next, to make a calculation relevant to the experiment, the $\mathrm{KH}$ instability in LAPD geometry is considered using the experimental density profile and a model $\phi_{0}(r)$ profile with amplitude values relevant to the experiment. The background potential profile is similar to expression (16), but the delta-functions in $\phi_{0}^{\prime \prime \prime}$ are replaced by Gaussians (exact expression is given in the Appendix C, Eq. (C1)). This calculation represents a strong test of the terms involving background flows in Eqs. (9-10) since some of these terms only contribute when both $\nabla N_{0}$ and $\nabla \phi_{0}$ exist. Note also that with non-constant $N_{0}(r)$, the density perturbation is not zero, unlike in the situation considered above. There is no analytic solution in this 
case, therefore we compare the BOUT solution with the results of the eigenvalue solver for the system of equations (144). The comparison is presented in Fig. 5, The result is similar to the previous KH case, with a cutoff in perpendicular wavenumber. In LAPD geometry, for this particular choice of profiles, this cutoff translates into $m_{\theta} \approx 8$; the $\mathrm{KH}$ mode is stable above this value. BOUT reproduces the direct eigenvalue solution with a very good accuracy of $\lesssim 2 \%$ for a 100 point radial grid size.

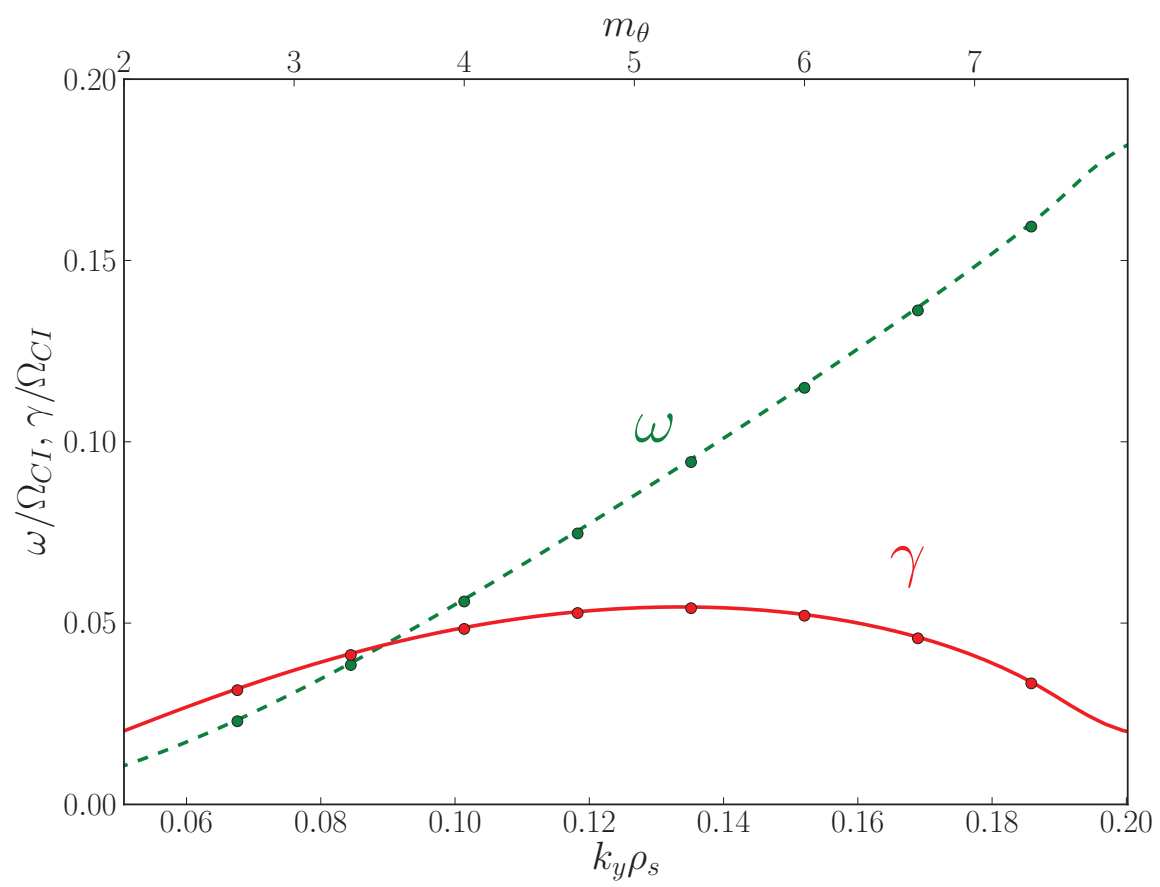

FIG. 5. (Color Online) Eigenvalues (frequency - dashed line, growth rate - solid line) of the Kelvin-Helmholtz instability as a function of perpendicular wavenumber. Circles - BOUT results. Cylindrical geometry, experimental density profile, LAPD plasma parameters.

\section{Interchange instability}

Strong azimuthal flows in LAPD not only affect the frequency of the waves through a Doppler shift, but can also modify the growth rate even for uniform rotation due to the induced centrifugal force. In this section the rotation-driven interchange mode is considered in the presence of background density gradient.

To separate the interchange mode from the other instabilities, the parallel wave number 
is set to zero (this removes the drift wave branch of the dispersion relation) and a uniform rotation profile is chosen with normalized rotation frequency $\Omega: \phi_{0}=\phi_{x} r^{2} / r_{\text {max }}^{2}=\Omega r^{2} / 2$ (this removes the $\mathrm{KH}$ mode). Assuming an exponential equilibrium density profile with gradient scale length $L_{n}$, Eq. (6) is written as

$$
\phi^{\prime \prime}+\frac{1}{r} \phi^{\prime}-\frac{1}{L_{n}} \phi^{\prime}-\frac{1}{r L_{n}}\left(\frac{d^{2}}{\tilde{\omega}^{2}}+\frac{2 d}{\tilde{\omega}}\right) \phi-\frac{m^{2}}{r^{2}} \phi=0
$$

where $d$ is the Doppler shift, $d=m \phi_{0}^{\prime} / r=2 m \phi_{x} / r_{\text {max }}^{2}=m \Omega, \tilde{\omega}=\omega-d$, and $L_{n}=N_{0} / N_{0}^{\prime}$.

There are two tractable limits where analytic solution can be found, $k L_{n} \gg 1$ (slow variation), and $k L_{n} \ll 1$ (sharp interface).

For small density gradient, the $1 / L_{n}$ term can be dropped when compared with the $1 / r$ term. Employing a change of variable $x=\sqrt{r}$, Eq. (19]) is rewritten as Bessel's equation:

$$
x^{2} \phi^{\prime \prime}(x)+x \phi^{\prime}(x)+\left(4 C^{2}-4 m^{2} x^{2}\right) \phi(x)=0
$$

where $C^{2}=-\frac{1}{L_{n}}\left(\frac{d^{2}}{\tilde{\omega}^{2}}+\frac{2 d}{\tilde{\omega}}\right)$. The solution is given as a sum of Bessel functions of the first and the second kind:

$$
\phi(x)=C_{1} J_{2 m}(-2 C x)+C_{2} Y_{2 m}(-2 C x) .
$$

The dispersion relation is obtained by imposing the boundary conditions $\phi\left(r_{\min }\right)=\phi\left(r_{\max }\right)=$ 0 on this function. For simplicity $r_{\min }=0$ is chosen. $Y_{2 m}(x)$ diverges at the axis, so the dispersion relation in this case is given by the condition

$$
J_{2 m}\left(-2 C \sqrt{r_{\max }}\right)=0
$$

For large $m_{\theta}$ the position of the first zero of the Bessel function $J_{m}(x)$ can be estimated ${ }^{24}$ as $m_{\theta}$ (e.g. $j_{m}=36.1$ for $m=30$ and the relative error monotonically decreases for larger $\left.m_{\theta}\right)$. This results in a simple approximate equation for the interchange eigenmode:

$$
-C \sqrt{r_{\max }}=m
$$

which yields the approximate dispersion relation (again using $L_{n} \gg r_{\max }$ )

$$
\omega=m \Omega \pm i \Omega \sqrt{\frac{r_{\max }}{L_{n}}}
$$

Note that the growth rate $\gamma=\Omega \sqrt{r_{\max } / L_{n}}$ can be obtained from the well known dispersion relation of the Rayleigh-Taylor instability driven by gravity, $\gamma \sim \sqrt{g / L_{n}}$, if gravity is replaced by the centrifugal force of the rotation, $g=\Omega^{2} r_{\max }$. 

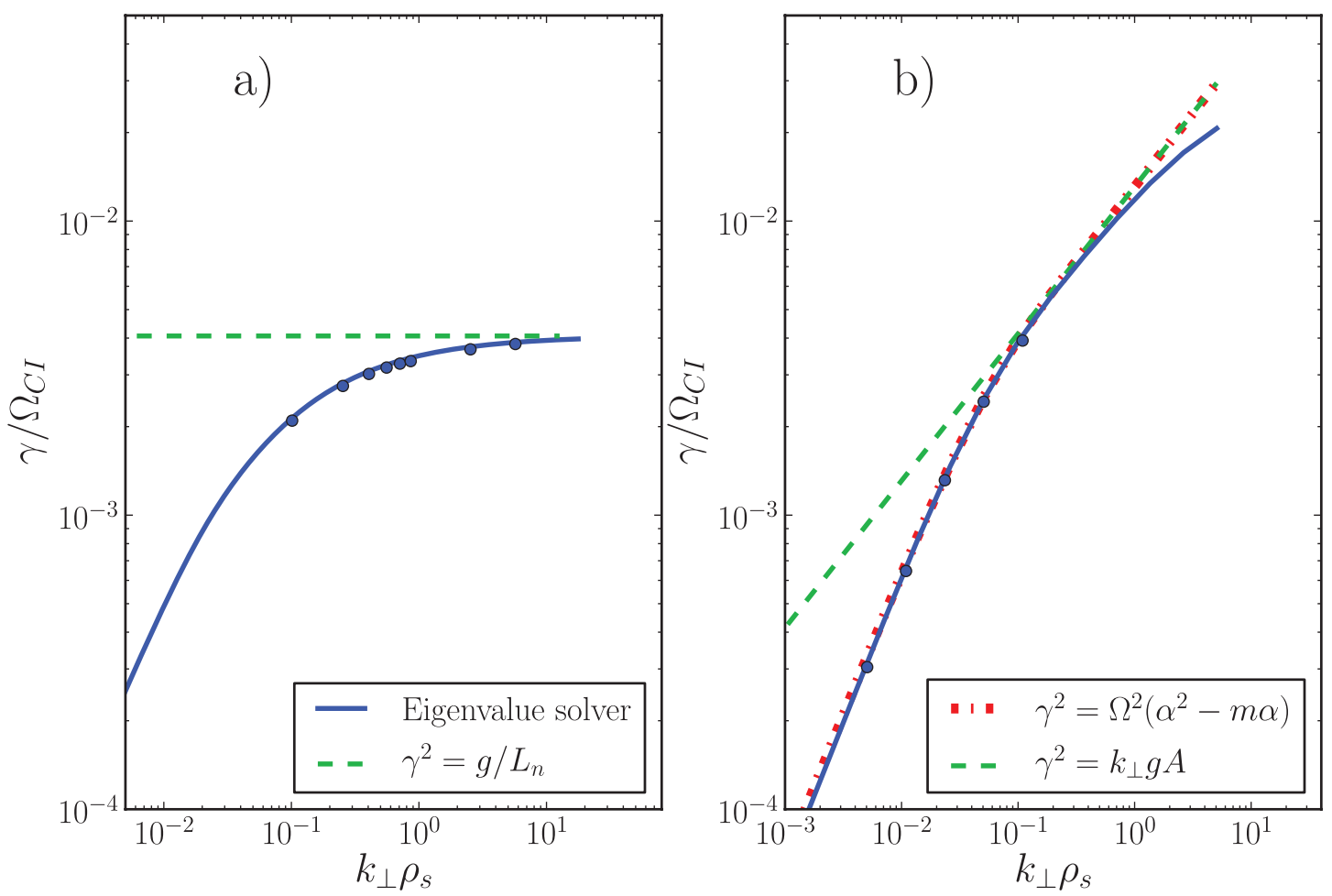

FIG. 6. (Color Online) Interchange mode in a rotating cylinder for the case of exponential density profile (a) and piecewise-constant density (b). Solid line - eigenvalue solution, dashed line asymptotic at large $k_{\perp}$, dashdot line - exact analytic solution for case (b), circles - BOUT results.

The growth rate given by Eq. (24) is independent of $m_{\theta}$ and represents an asymptotic solution for large $m_{\theta}$. This asymptotic solution and the exact solution of Eq. (19) are shown in Fig. 6(a).

Another limit where a simple analytic solution can be found is the case of a piecewiseconstant density profile with a sharp interface, $N_{0}(r)=N_{1}$ for $r<r_{0}$ and $N_{0}(r)=N_{2}$ for $r>r_{0}, r_{0}=r_{\max } / 2, N_{1}>N_{2}$. Eq. (19) at $r \neq r_{0}$ then becomes

$$
\phi^{\prime \prime}+\frac{1}{r} \phi^{\prime}-\frac{m^{2}}{r^{2}} \phi=0
$$

with the general solution $\phi(r) \sim r^{ \pm m}$. Matching the values of $\phi(r)$ at the interface, applying the boundary condition at the conducting shell $\phi\left(r_{\max }\right)=0$, and integrating Eq. (19) in a small region near the interface to account for the jump in $N_{0}$ and $\phi^{\prime}$, we obtain the dispersion 
relation

$$
\tilde{\omega}=\Omega\left(-\alpha \pm \sqrt{\alpha^{2}-m \alpha}\right)
$$

where $\alpha=A\left(2^{2 m}-1\right) /\left(2^{2 m}-A\right)$ and $A$ is the Atwood number $A=\left(N_{1}-N_{2}\right) /\left(N_{1}+N_{2}\right)$.

In the limit of large $m_{\theta}$ cylindrical effects become insignificant and the growth rate converges to that of the gravity-driven Rayleigh-Taylor instability in a slab for two fluids with sharp interface, $\gamma \approx \sqrt{\Omega^{2} m A}=\sqrt{k g A}$. The exact solution and the asymptotic solution at large $m_{\theta}$ are shown in Fig. 6(b). The solid line represents the eigenvalue solution of the system (5) where the piecewise-constant density profile is approximated with tanh function. At higher $m_{\theta}$ numbers (here, at $m \gtrsim 20$, or $k_{\theta} \rho_{s} \gtrsim 1$ ) the finite width of the interface region becomes important compared to $1 / k_{\theta}$, so the numerical (eigenvalue) solution starts to deviate from the analytic solution (26).
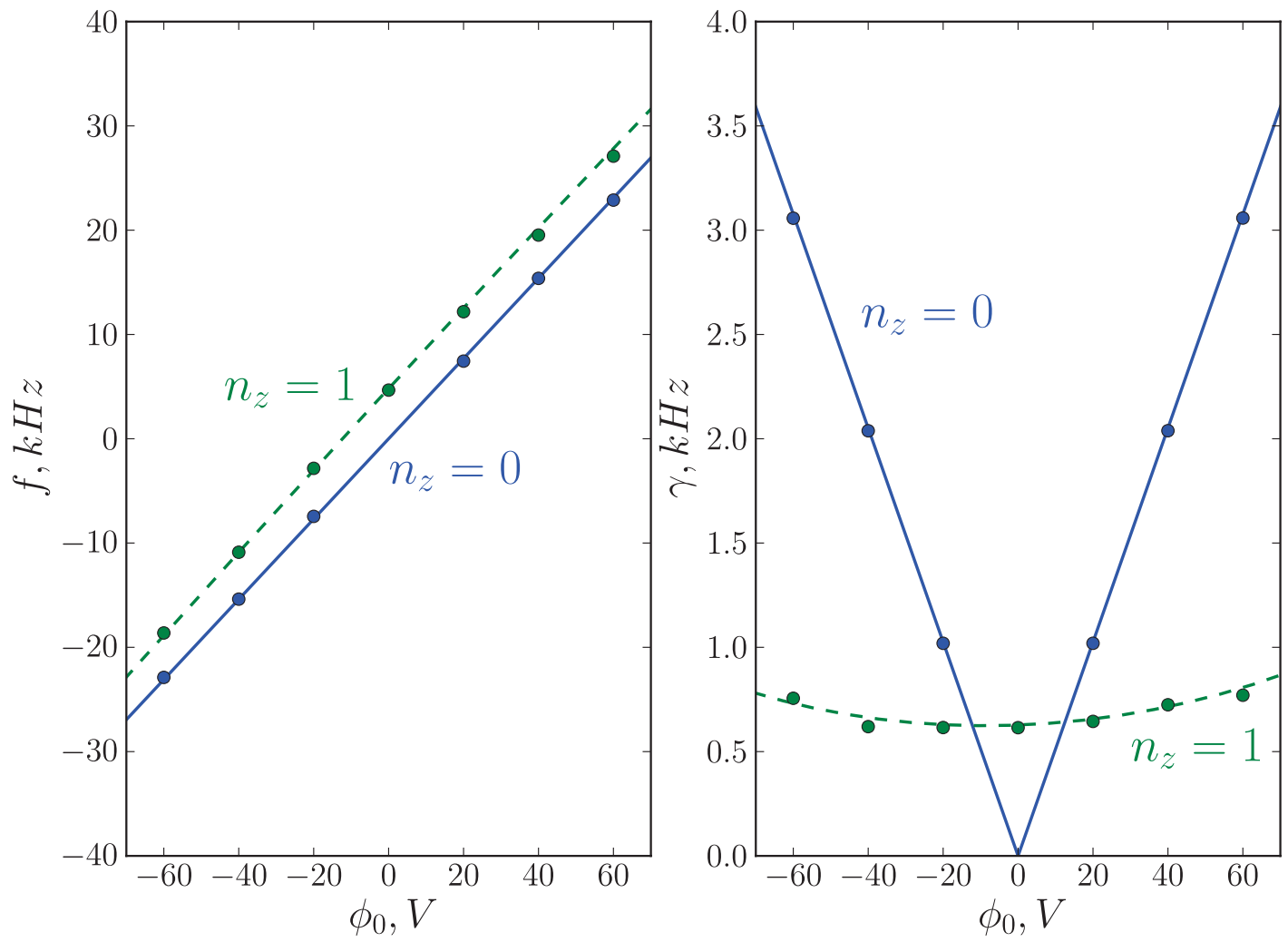

FIG. 7. (Color Online) Interchange mode $\left(k_{\|}=0\right)$ destabilized by uniform rotation and driftinterchange mode $\left(k_{\|}=2 \pi / L\right)$.

The system of time-evolution equations used in BOUT to reproduce the interchange mode 
can be obtained from Eqs. (7f-9) by setting $k_{\|}=0$ :

$$
\begin{aligned}
& \partial_{t} N=-\mathbf{v}_{\boldsymbol{E}} \cdot \nabla N \\
& \partial_{t} \varpi=-\mathbf{v}_{\boldsymbol{E}} \cdot \nabla \varpi+\mathbf{b} \times \nabla N \cdot \nabla v_{E}^{2} / 2-\nu_{i n} \varpi
\end{aligned}
$$

where all variables $\left(N, \mathbf{v}_{\boldsymbol{E}}, \varpi\right)$ contain both the equilibrium part and the fluctuating component. These equations are linearized in BOUT, and solved with the same parameters as used in the two analytic examples discussed above (Fig. 6). BOUT simulation recovers the interchange mode solution for both limits (slowly varying exponential and piecewise constant profiles) which verifies the correct implementation of the new terms involving background flows in BOUT.

In order to investigate the effect of uniform rotation on the interchange and driftinterchange instabilities in LAPD plasmas, a configuration with experimental density profile and $\phi_{0}(r) \sim r^{2}$ is considered. The results of this calculation are presented in Fig. (77) as a function of rotation velocity. Two axial harmonics are shown: $n_{z}=0$ (pure interchange mode) and $n_{z}=1$ (drift-interchange instability). At $\phi_{0}=0 \mathrm{~V}, n_{z}=1$ branch corresponds to a pure drift mode. As $\phi_{0}$ increases, the frequency of this mode is Doppler-shifted and the growth rate is modified by the centrifugal force. At large rotation velocities, the disparity between the large real part of the frequency and the small growth rate is hard to resolve numerically using an initial-value code, so the BOUT results slightly deviate from the direct eigenvalue solution of the dispersion relation.

\section{LINEAR INSTABILITIES IN LAPD}

Now that simple analytic solutions for each of the instabilities supported by Eqs. (144) have been presented, linear instabilities for LAPD parameters and experimental profiles will be considered and the growth rates for the different mode branches will be compared.

Fig. (8) shows the growth rates and frequencies of the $\mathrm{KH}$, drift and interchange modes for LAPD parameters using experimentally measured density profiles. The complete set of parameter values including the polynomial fit of the experimental density profile is shown in Appendix C. Three different model background potential profiles are chosen here to separate the instability branches: same profile as used in Fig. 5 for the KH mode (given by Eq. (C1) ), uniform rotation profile $\phi_{0}(r) \sim r^{2}$ for the interchange mode, and zero potential 

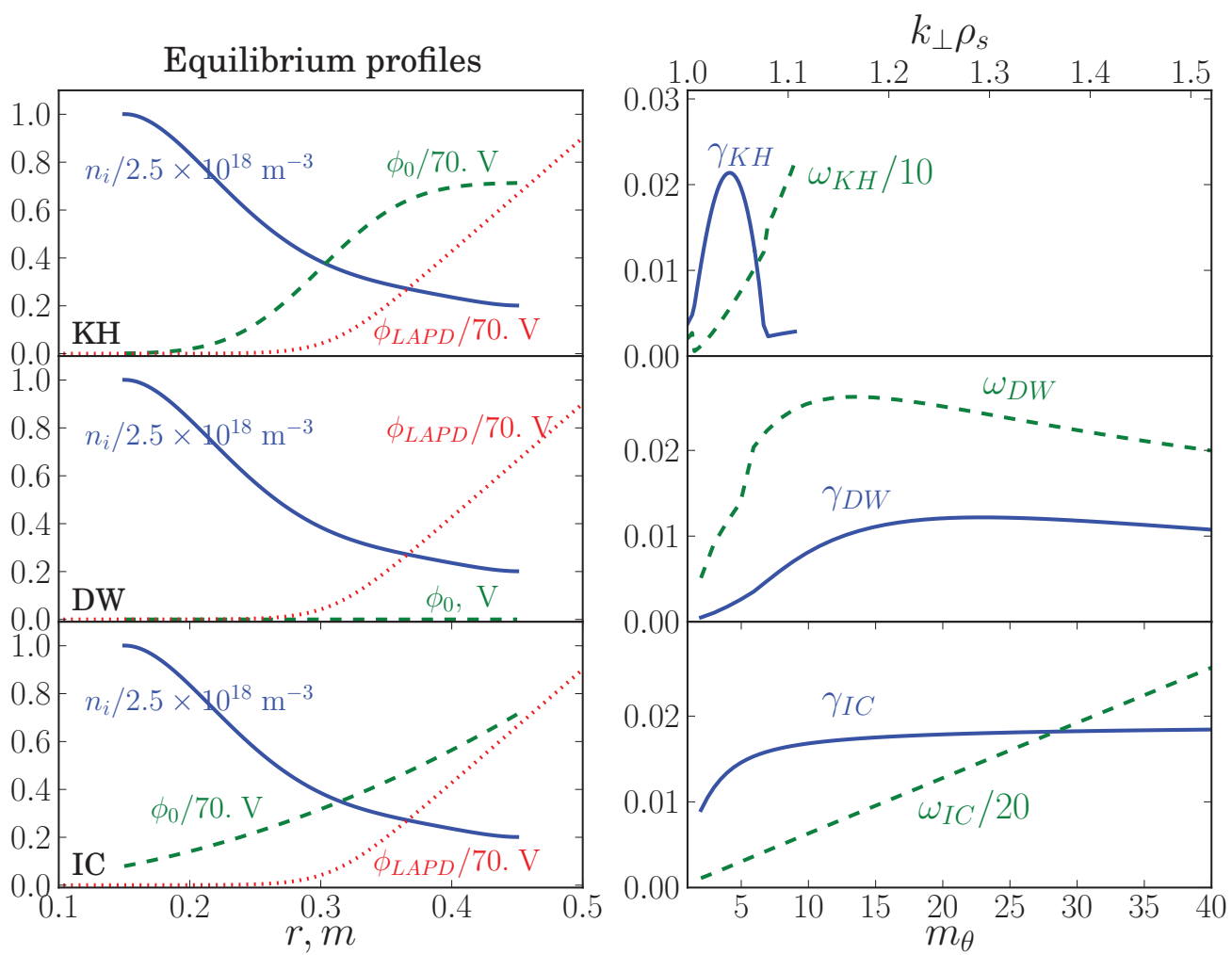

FIG. 8. (Color Online) Kelvin-Helmholtz, drift and interchange branches of the dispersion relation for LAPD parameters as a function of azimuthal mode number. Left: equilibrium profiles, right: frequency and growth rate of the instability. For KH and IC $n_{z}=0$, for DW $n_{z}=0.5$. For DW case $\phi_{0}(r)=0$, for IC instability $\phi_{0}(r) \sim r^{2}$ (uniform rotation), for $\mathrm{KH}$ mode $\phi_{0}(r)$ is given by Eq. (C1). As a reference, the experimentally measured $\phi_{L A P D}$ profile is shown in dotted line (left).

for the drift wave instability. The magnitude of the radial potential drop in the $\mathrm{KH}$ and IC cases is of the same order as the measured value in biased discharge experiments ${ }^{22}$. Even though a direct comparison of the three solutions is not possible because the background flow profiles and axial mode numbers are not the same, it is still informative to note that the growth rates of all three branches of instability are of similar magnitude. Therefore, all three instabilities can potentially compete in LAPD plasmas.

Similar results are observed in a calculation of linear growth rates when using selfconsistent, experimentally measured profiles of density, electron temperature and flow (Fig. 9). Two cases are considered here, biased and unbiased plasma discharges (with and without bias-driven azimuthal edge flow $)^{22}$. In the unbiased configuration, the az- 

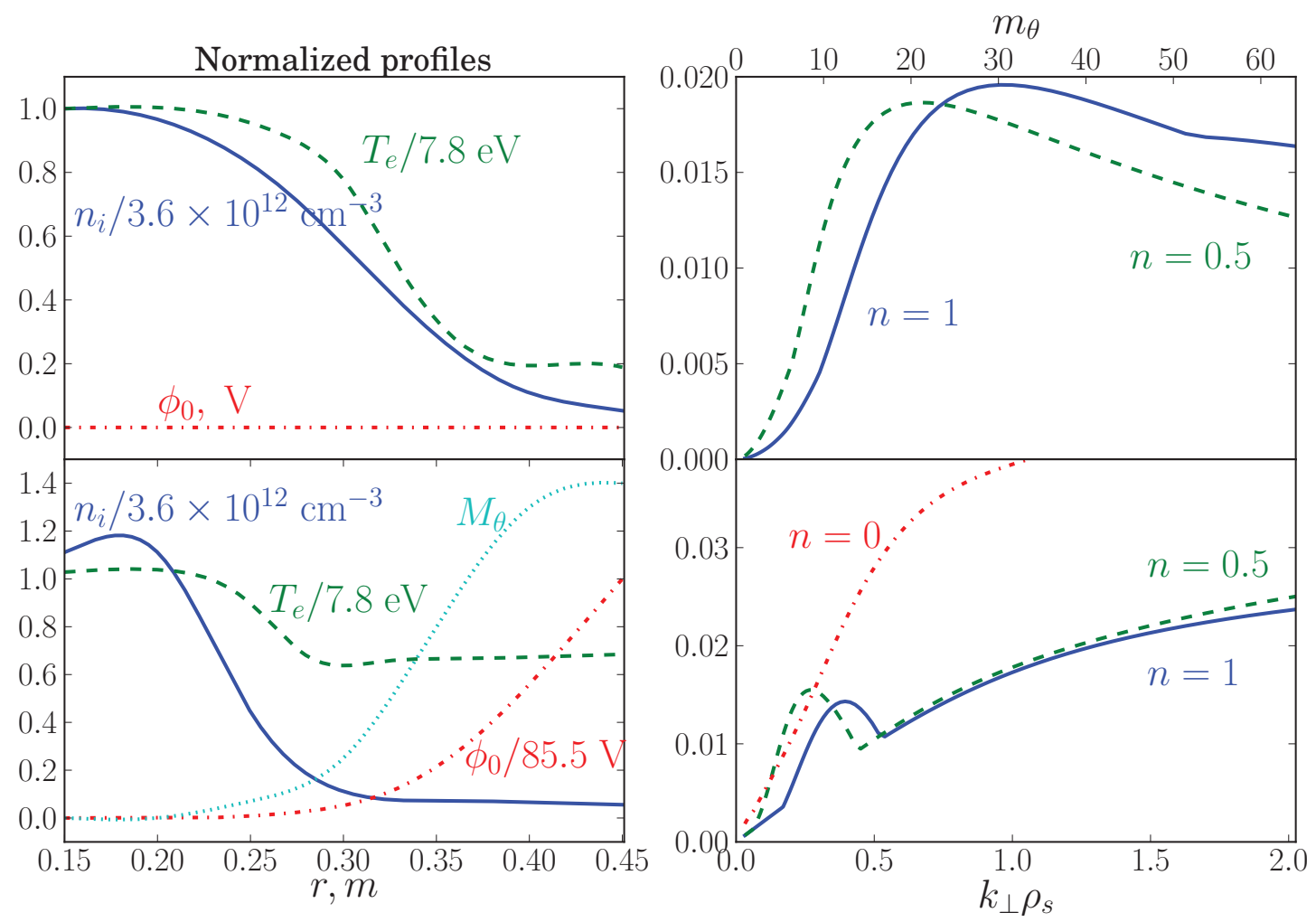

FIG. 9. (Color Online) Equilibrium profiles of the density, electron temperature, potential and Mach number (left) and the fastest growth rates (right) of the perturbation with axial mode number $n_{z}=0,0.5,1$. Top: unbiased LAPD discharge. Bottom: LAPD discharge with applied radial bias. Experimental data taken from Maggs et al..$^{22}$.

imuthal flow values are much smaller than in the biased case, so we use zero azimuthal flow for this calculation. In the unbiased case (Fig. 9, top), only the drift wave branch is present, with comparable maximum growth rates for $n_{z}=1$ and $n_{z}=0.5$. In the biased case (Fig. 9, bottom), the growth rates at $m_{\theta} \lesssim 10$ for the three harmonics $n_{z}=0, n_{z}=0.5$ and $n_{z}=1$ are comparable. From the eigenfunction analysis, it can be concluded that $n_{z}=1$ harmonic is predominantly interchange at $m_{\theta} \lesssim 5$, then drift wave-like at $5 \lesssim m_{\theta} \lesssim 17$ and again IC-like at higher $m_{\theta}$. An example of the eigenfunctions of the potential perturbation for the biased case is shown in Fig. 10. At $m_{\theta}=3$ and $m_{\theta}=20$, the axial mode $n_{z}=1$ is localized near the edge of the plasma where the azimuthal flows are strongest (see $M_{\theta}$ profile in Fig. 9, bottom), which is consistent with the rotational interchange instability. At $m_{\theta}=12$, the $n_{z}=1$ harmonic eigenfunction is localized near $r \sim 28 \mathrm{~cm}$, where the gradients of the density and electron temperature are strongest, which indicates the drift-wave-like character 
of the mode.

The real frequencies of these modes are consistent with experimental observation; in the unbiased case, at the peak of the growth rate for $n_{z}=0.5(m \sim 20)$ the mode frequency is $f=4.7 \mathrm{kHz}$ which is in the heart of the measured broadband fluctuation spectrum in unbiased plasmas, although a lower mode number would be consistent with the measured correlation function ${ }^{10}$. In the biased case, the local maximum of the growth rate of $n_{z}=0.5$ mode is at $m_{\theta}=8$, which is also consistent with measured LAPD value of $m_{\theta} \lesssim 10^{10}$. Higher growth rates at large $m_{\theta}$ might not be relevant when viscosity effects are included in the calculation, since high $k_{\perp}$ modes will be damped by viscosity. The computed linear eigenfunctions are consistent with the observed fluctuation profiles in the unbiased case, localized to the density gradient region. In the biased case, eigenfunctions localized to the region of strong density gradient are found as well as flow-driven modes that are localized to the far edge away from the strong gradient region. The latter is consistent with the observation of increased electric field fluctuations in the far-edge plasma with increased bias (see Fig. 9c of Ref. 10). The linear prediction that Kelvin-Helmholtz and/or rotational interchange might be the dominant instabilities in the biased case could also be consistent with measurements of the cross phase between density and electric field fluctuations. In going from unbiased to biased plasmas in LAPD, a dramatic change in the cross phase is observed, which could be consistent with a change in the dominant instability $\underline{10}$.

A detailed comparison with the experimental data requires nonlinear analysis and simulation, which is the subject of a companion paper ${ }^{15}$. However, it is still illustrative to apply quasilinear theory or mixing length $\operatorname{arguments}^{25,26}$ using the linear calculation results presented above. For drift waves driven by the background density gradient the mixing length estimate assumes that the saturation is reached when the perturbed gradients become comparable to the equilibrium gradients:

$$
n k_{\perp} \sim n_{0} / L_{n}
$$

so that

$$
n / n_{0} \sim q \phi / T_{e} \sim 1 / k_{\perp} L_{n}
$$

The weak turbulence theory modifies this estimate by a factor $\sqrt{\gamma / \omega_{*}}$ :

$$
n / n_{0} \sim q \phi / T_{e} \sim \sqrt{\gamma / \omega_{*}} / k_{\perp} L_{n}
$$




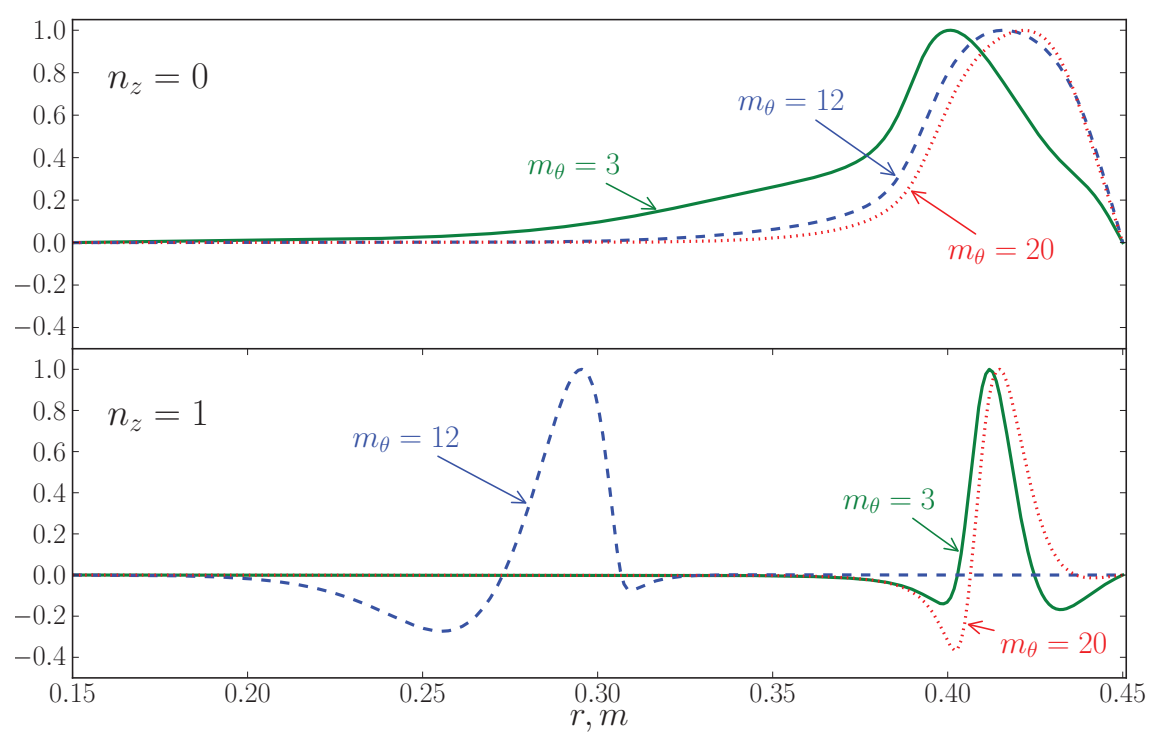

FIG. 10. (Color Online) Eigenfunctions of the perturbed potential in the biased plasma configuration (case shown in Fig. 9, bottom) for axial mode number $n_{z}=0,1$, azimuthal harmonics $m_{\theta}=3,12,20$.

where $\gamma$ and $\omega_{*}$ are the growth rate and frequency of the fastest linear mode in the system.

In the unbiased case (Fig. 9, top), the maximum growth rate is achieved for modes with $m_{\theta} \sim 20-30$, so $k_{\perp} \sim m_{\theta} / r \sim 100 \mathrm{~m}^{-1}$. The frequency is close to the growth rate for these modes, so $\gamma / \omega_{*} \sim 0.5-1$. The background density gradient scale length near the radial location of interest (cathode edge at $\sim 28 \mathrm{~cm}$ where the equilibrium density gradient is mostly localized) is $L_{n}=n_{0} / n_{0}^{\prime} \sim 0.1 \mathrm{~m}$. Therefore, both the simple mixing length argument and the weak turbulence theory give a similar estimate for the saturated level of turbulence, $n / n_{0} \sim q \phi / T_{e} \sim 10 \%$. This estimate is close to the observed amplitude of fluctuations in LAPD measurements and in the nonlinear simulations of LAPD discharge $\underline{10}, 15$. The diffusion coefficient estimate based on the mixing length argument, $D \sim \gamma / k_{\perp}^{2} \sim 2 \mathrm{~m}^{2} / \mathrm{s}$, is close to the value calculated from a saturated state in a self-consistent nonlinear simulation $D \sim 3 \mathrm{~m}^{2} / \mathrm{s}^{15}$. This value is comparable to Bohm diffusion, $D_{B} \sim 8 \mathrm{~m}^{2} / \mathrm{s}$, and diffusive transport with a Bohm diffusion coefficient has been found to describe the measured profiles well in the unbiased case $^{22}$.

The biased configuration has reduced radial transport due to strong azimuthal flows and is better described by classical diffusion coefficient ${ }^{22}$. Detailed analysis of this case requires 
a self-consistent nonlinear simulation that takes into account the average radial electric field profile; this will be the subject of future work.

\section{EFFECT OF ION-NEUTRAL COLLISIONS}

The results presented in previous sections do not include ion-neutral collision terms that enter the vorticity equation Eq. (9). The general effect of the $\nu_{i n}$ term is to damp the vorticity perturbations (as can be seen from Eq. (9)) and to stabilize the wave. In Fig. 11, variation of the real frequency and growth rates for the three modes (drift, KH, and IC) is shown as a function of the ion-neutral collisionality parameter $\nu_{i n}$. Each branch is taken at a fixed azimuthal mode number $m_{\theta}$ that corresponds to the maximum growth rate without neutrals (same solution as in Fig. 8), except for the interchange branch, where $m_{\theta}=10$ is chosen). All of the frequencies and growth rates are normalized to the corresponding values at $\nu_{\text {in }}=0$.

When ion-neutral collisions are included, the drift wave growth rate decreases and the mode can be completely stabilized at sufficiently high neutral density. For a typical LAPD discharge, the rough estimate of $n_{N}$ is $\sim 5 \times 10^{11} \mathrm{~cm}^{-3} \underline{22}$ which translates into $\nu_{\text {in }} \sim$ $2 \times 10^{-3} \Omega_{i}$. At these values of $n_{N}$, the effect of the neutrals on the linear stability is relatively weak. To completely stabilize the drift mode, $n_{N}$ should be larger by a factor of 10 (Fig. 11, red). However, due to significant uncertainty in the values of neutral density in LAPD, ion-neutral collisions can potentially be important. More importantly, initial nonlinear simulations using BOUT show that even at the values near the estimated $\nu_{i n} \sim$ $2 \times 10^{-3} \Omega_{i}$, the neutral damping is important for the dynamics of the self-generated zonal flows 15 .

Compared to the drift mode, the $\mathrm{KH}$ instability is more strongly affected by the ionneutral collisions. Compared to the neutral-free case, at the estimated for LAPD level of ion-neutral collisions, the growth rate drops by $\sim 35 \%$ and the mode is completely stabilized at $\nu_{\text {in }} / \Omega_{i} \sim 0.006$.

The interchange mode turns out to be weakly affected by ion-neutral collisions. For all three instability branches, the frequency of the mode remains nearly constant in the range of relevant values of neutral collisionality (Fig. 11, dashed lines). 


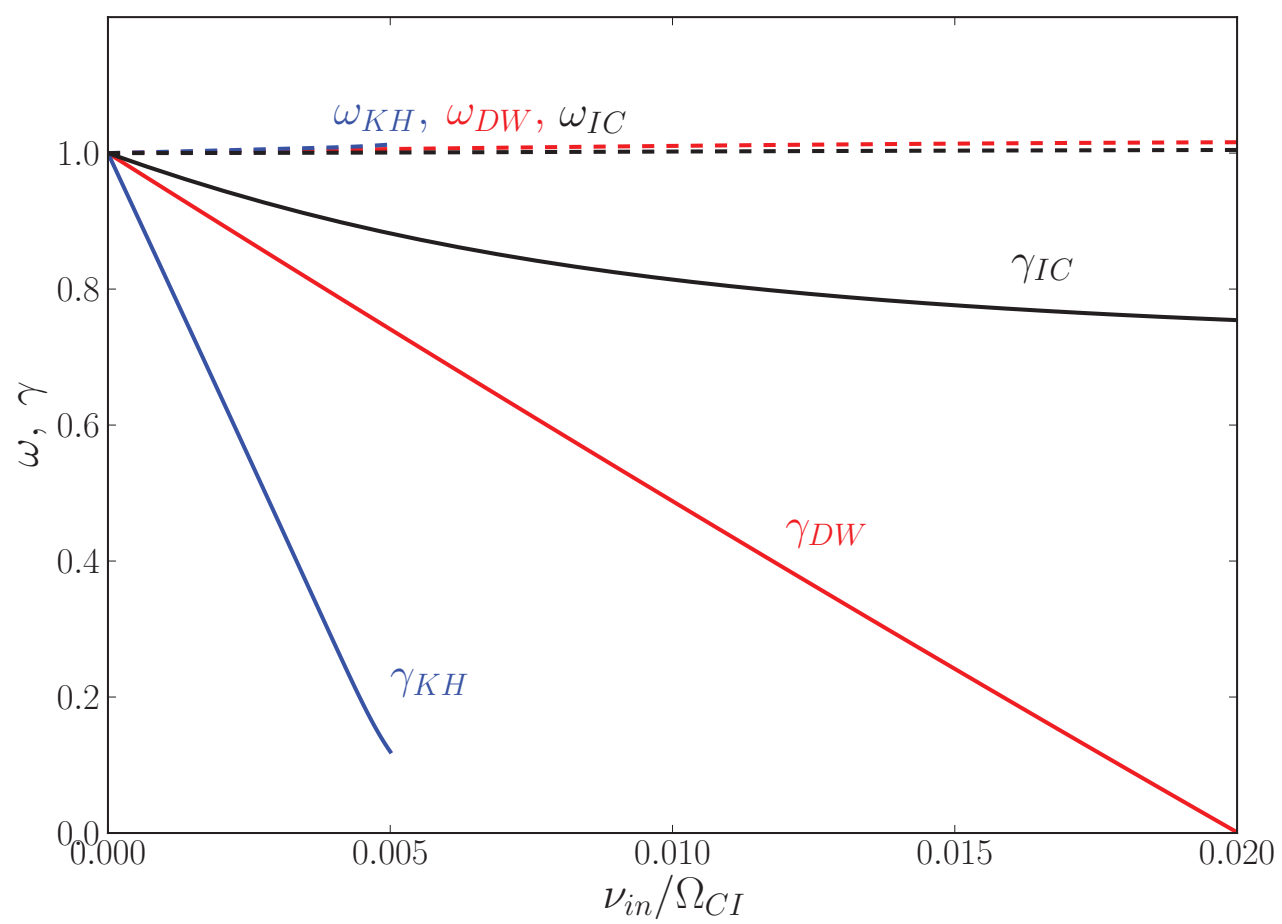

FIG. 11. (Color Online) Effect of ion-neutral collisions on the Kelvin-Helmholtz (KH), drift wave (DW) and interchange (IC) branches of the dispersion relation. All scans are normalized to the corresponding value at $\nu_{i n}=0$. Solid line - growth rate, dashed - frequency. Estimate for typical LAPD parameters: $\nu_{i n} / \Omega_{i} \sim 2 \times 10^{-3}$.

\section{CONCLUSIONS}

The 3D initial value fluid code for tokamak edge plasma has been adapted to LAPD geometry. A separate eigenvalue solver for BOUT set of linearized equations has been developed for an independent verification of BOUT results when an analytic solution is not available. Background flow terms have been added to BOUT equations to allow simulation of flow-driven instabilities. Periodic boundary conditions has been adopted in the parallel direction as a first step. A more realistic model of sheath boundary conditions will be implemented in future simulations to capture the effect of the parallel boundary on the dynamics of the average radial electric field.

Starting from a system of 3D plasma fluid equations, the derivation of a dispersion relation is presented that includes three plasma instability branches: resistive drift mode, Kelvin- 
Helmholtz mode, and interchange mode; the latter two driven by plasma azimuthal flow. It is demonstrated that for LAPD parameters the growth rates for all three branches may be comparable, so all three physical mechanisms are potentially important. Interaction with neutrals, for the estimated LAPD neutral density, does not significantly affect the linear stability of considered modes. However, neutral dynamics can be important for the zonal flow generation in nonlinear simulations.

The initial value solution obtained with BOUT accurately reproduces analytic calculations of the properties of the three instabilities, including growth rates, frequencies and eigenfunctions. The code solution is in full agreement with analytic and eigenvalue solutions, for both model profiles and experimentally relevant profiles, which lends confidence for proceeding with nonlinear simulations and validation of BOUT against LAPD measurements. Aspects of these linear theoretical estimates (dominant mode numbers, mode frequency, and quasilinear estimates of fluctuation amplitudes and diffusion coefficient) are consistent with the experimental measurements in LAPD. However, more detailed comparison with experiment requires self-consistent nonlinear simulations and this work is underway. Initial nonlinear calculations based on the model discussed in this work, and detailed comparisons with experimental data, will be presented in a companion paper ${ }^{15}$.

\section{ACKNOWLEDGMENTS}

This work was supported by DOE Fusion Science Center Cooperative Agreement DEFC02-04ER54785, NSF Grant PHY-0903913, and by LLNL under DOE Contract DE-AC5207NA27344. BF acknowledges support through appointment to the Fusion Energy Sciences Fellowship Program administered by Oak Ridge Institute for Science and Education under a contract between the U.S. Department of Energy and the Oak Ridge Associated Universities.

\section{Appendix A: Derivation of fluid equations}

The perpendicular component of the current in Eq. (4) is found from the fluid equation for the ions Eq. (3). Note that the viscosity tensor $\Pi$ and ion pressure terms are dropped here, since we neglect the ion temperature effects in this work. Solving it for ion velocity $\mathbf{v}_{i}$ 
in the Lorentz term, we obtain $\mathbf{v}_{i \perp}$ as a sum of the $\mathbf{E} \times \mathbf{B}$, polarization and Pedersen drifts:

$$
\mathbf{v}_{i \perp}=\mathbf{v}_{E}+\mathbf{v}_{p i}+\mathbf{v}_{f i}
$$

where $\mathbf{v}_{E}=c \mathbf{E} \times \mathbf{B} / B^{2}, \mathbf{v}_{p i}=\frac{1}{\Omega_{i}}\left(\partial_{t}+\mathbf{v}_{i} \cdot \nabla\right) \mathbf{v}_{i}, \mathbf{v}_{f i}=\frac{\nu_{i n}}{\Omega_{i}} \mathbf{B} \times \mathbf{v}_{i} / B$.

The main contributions to the perpendicular part of the current divergence come from the ion polarization current (the electron olarization drift is smaller by mass ratio) and Pedersen current:

$$
\begin{aligned}
\nabla \cdot \mathbf{J}_{\perp} \approx \nabla \cdot\left(e n \mathbf{v}_{p i}+e n \mathbf{v}_{f i}\right) \\
=\frac{1}{\Omega_{i}} \nabla \cdot\left\{e n \mathbf{b} \times\left(\frac{\partial \mathbf{v}_{i}}{\partial t}+\mathbf{v}_{i} \cdot \nabla \mathbf{v}_{i}\right)+e n \nu_{i n} \mathbf{b} \times \mathbf{v}_{i}\right\} \\
\quad \approx \frac{1}{\Omega_{i}} \nabla \cdot\left\{e n \mathbf{b} \times\left(\partial_{t}+\mathbf{v}_{E} \cdot \nabla\right) \mathbf{v}_{E}+e n \nu_{i n} \mathbf{b} \times \mathbf{v}_{E}\right\}
\end{aligned}
$$

To make the linear expansion of the current continuity equation exactly equivalent to the linearized BOUT vorticity equation discussed below, we employ the approximation $\nabla$. $\left(n \mathbf{v}_{i}\right) \sim \mathbf{v}_{E} \cdot \nabla n$ (well satisfied for typical LAPD parameters), the same way it is done in previous work by Simakov and Catto $\underline{20}$ (Eq. D3):

$$
\nabla \cdot \mathbf{J}_{\perp} \approx-\frac{m_{i} c^{2}}{B^{2}} \nabla \cdot\left\{\left(\partial_{t}+\mathbf{v}_{E} \cdot \nabla\right)\left(n \nabla_{\perp} \phi\right)+\nu_{i n} n \nabla_{\perp} \phi\right\}
$$

Substituting this expression in the charge conservation equation Eq. (4), combining with the continuity equation Eq. (11) and parallel projection of the electron momentum equation Eq. (2) and linearizing, we obtain:

$$
\begin{aligned}
& \partial_{t} N+\mathbf{b}_{\mathbf{0}} \times \nabla_{\perp} \phi_{0} \cdot \nabla N=-\mathbf{b}_{\mathbf{0}} \times \nabla_{\perp} \phi \cdot \nabla N_{0}-N_{0} \nabla_{\|} v_{\| \mathrm{e}} \\
& \partial_{t} v_{\| \mathrm{e}}+\mathbf{b}_{\mathbf{0}} \times \nabla_{\perp} \phi_{0} \cdot \nabla v_{\| \mathrm{e}}=-\mu \frac{T_{e 0}}{N_{0}} \nabla_{\|} N+\mu \nabla_{\|} \phi-\nu_{e} v_{\| \mathrm{e}} \\
& N_{0} \nabla_{\|} v_{\| \mathrm{e}}=-\nabla_{\perp} \cdot\left(N_{0} \partial_{t} \nabla_{\perp} \phi+\partial_{t} N \nabla_{\perp} \phi_{0}\right. \\
&+\mathbf{b}_{\mathbf{0}} \times \nabla_{\perp} \phi_{0} \cdot \nabla\left(N_{0} \nabla_{\perp} \phi_{0}\right) \\
&+\mathbf{b}_{\mathbf{0}} \times \nabla_{\perp} \phi_{0} \cdot \nabla\left(N_{0} \nabla_{\perp} \phi\right) \\
&+\mathbf{b}_{\mathbf{0}} \times \nabla_{\perp} \phi \cdot \nabla\left(N_{0} \nabla_{\perp} \phi_{0}\right) \\
&+\mathbf{b}_{\mathbf{0}} \times \nabla_{\perp} \phi_{0} \cdot \nabla\left(N \nabla_{\perp} \phi_{0}\right) \\
&\left.+N_{0} \nu_{i n} \nabla_{\perp} \phi_{0}+N_{0} \nu_{i n} \nabla_{\perp} \phi+N \nu_{i n} \nabla_{\perp} \phi_{0}\right)
\end{aligned}
$$

We project these equations on cylindrical coordinates $(r, \theta, z)$ and assume the fluctuations are of the form $f(\mathbf{x})=f(r) \exp \left(i m_{\theta} \theta+i k_{\|} z-i \omega t\right)$. Solving the first two equations for $N$ and 
$v_{\| \mathrm{e}}$, and substituting them in the current equation, we obtain $1 \mathrm{D}$ equation for the perturbed potential:

$$
\begin{gathered}
C_{2}(r) \phi^{\prime \prime}+C_{1}(r) \phi^{\prime}+C_{0}(r) \phi=0, \\
C_{2}(r)=\left(\nu_{i n}-i \tilde{\omega}\right) \\
C_{1}(r)=\left(\nu_{i n}-i \tilde{\omega}\right)\left(\frac{1}{r}-\frac{1}{L_{n}}+\phi_{0}^{\prime} \lambda_{N}\right)+i m_{\theta} \frac{1}{r L_{n}} \phi_{0}^{\prime} \\
C_{0}(r)=\left(\nu_{i n}-i \tilde{\omega}\right)\left(-\frac{m_{\theta}^{2}}{r^{2}}+\lambda_{N} \phi_{0}^{\prime}\left(\frac{1}{r}-\frac{1}{L_{n}}\right)+\left(\lambda_{N} \phi_{0}^{\prime}\right)^{\prime}\right) \\
+\frac{i m_{\theta}}{r^{3}}\left(\phi_{0}^{\prime}-r \phi_{0}^{\prime \prime}-r^{2} \phi_{0}^{\prime \prime \prime}-\frac{r}{N_{0}}\left(r N_{0}^{\prime} \phi_{0}^{\prime}\right)^{\prime}+\frac{r^{2}}{L_{n}} \phi_{0}^{\prime \prime}\right) \\
+i k_{\|} \lambda_{v}+i m_{\theta} \frac{1}{r} \lambda_{N} \phi_{0}^{\prime} \phi_{0}^{\prime \prime},
\end{gathered}
$$

where

$$
\begin{aligned}
\lambda_{v}(r, \tilde{\omega}) & =i k_{\|} \mu \frac{1-\frac{T_{e 0}}{\tilde{\omega} L_{n}} \frac{m_{\theta}}{r}}{\nu_{e}-i \tilde{\omega}+i k_{\|}^{2} \mu \frac{T_{e 0}}{\tilde{\omega}}} \\
\lambda_{N}(r, \tilde{\omega}) & =\frac{i k_{\|}^{2} \mu+\frac{m_{\theta}}{r} \frac{1}{L_{n}}\left(\nu_{e}-i \tilde{\omega}\right)}{\tilde{\omega}\left(\nu_{e}-i \tilde{\omega}\right)+i k_{\|}^{2} \mu T_{e 0}} \\
L_{n} & =-\frac{N_{0}}{N_{0}^{\prime}}, \quad \tilde{\omega}=\omega-\frac{m_{\theta}}{r} \phi_{0}^{\prime}
\end{aligned}
$$

\section{Appendix B: Derivation of the vorticity equation}

Expanding the charge conservation equation $\nabla \cdot \mathbf{J}=0$ as described in section II, we can write

$$
0=\nabla \cdot \mathbf{J}_{\|}+\nabla \cdot \mathbf{J}_{\perp}=\nabla_{\|}\left(N v_{\|}\right)-\nabla_{\perp} \cdot\left\{\left(\partial_{t}+\mathbf{v}_{E} \cdot \nabla\right)\left(n \nabla_{\perp} \phi\right)+\nu_{i n} n \nabla_{\perp} \phi\right\}
$$

Introducing the potential vorticity defined as $\varpi \stackrel{\text { def }}{=} \nabla_{\perp} \cdot\left(N \nabla_{\perp} \phi\right)$, we can rewrite the second term:

$$
\begin{aligned}
- & \nabla_{\perp} \cdot\left\{\left(\partial_{t}+\mathbf{v}_{E} \cdot \nabla\right)\left(N \nabla_{\perp} \phi\right)+\nu_{i n} n \nabla_{\perp} \phi\right\} \\
& =-\partial_{t} \varpi-\mathbf{v}_{E} \cdot \nabla \varpi-\nabla_{\perp} \mathbf{v}_{E}: \nabla_{\perp}\left(N \nabla_{\perp} \phi\right)-\nu_{i n} \varpi \\
& =-\partial_{t} \varpi-\mathbf{v}_{E} \cdot \nabla \varpi-\nabla_{\perp} \mathbf{v}_{E}: \nabla_{\perp} N \nabla_{\perp} \phi-N \nabla_{\perp} \mathbf{v}_{E}: \nabla_{\perp} \nabla_{\perp} \phi-\nu_{i n} \varpi
\end{aligned}
$$


The fourth term in this expression vanishes:

$$
\begin{aligned}
& \nabla_{\perp} \mathbf{v}_{E}: \nabla_{\perp} \nabla_{\perp} \phi=\nabla_{\perp}\left(\mathbf{b} \times \nabla_{\perp} \phi\right): \nabla_{\perp} \nabla_{\perp} \phi \\
= & \frac{1}{2}\left(\nabla_{\perp}^{2}\left(\nabla_{\perp} \phi \cdot \mathbf{b} \times \nabla_{\perp} \phi\right)-\left(\nabla_{\perp}^{2} \nabla_{\perp} \phi\right) \cdot\left(\mathbf{b} \times \nabla_{\perp} \phi\right)-\nabla_{\perp}^{2}\left(\mathbf{b} \times \nabla_{\perp} \phi\right) \cdot \nabla_{\perp} \phi\right) \\
= & \frac{1}{2}\left(-\left(\nabla_{\perp}^{2} \nabla_{\perp} \phi\right) \cdot\left(\mathbf{b} \times \nabla_{\perp} \phi\right)-\left(\mathbf{b} \times \nabla_{\perp} \nabla_{\perp}^{2} \phi\right) \cdot \nabla_{\perp} \phi\right)=0
\end{aligned}
$$

The third term in Eq. (B2) can be simplified as follows:

$$
\begin{aligned}
\nabla_{\perp} \mathbf{v}_{E}: \nabla_{\perp} N \nabla_{\perp} \phi & =\left\{\nabla_{\perp} \phi \cdot \nabla_{\perp}\left(\mathbf{b} \times \nabla_{\perp} \phi\right)\right\} \cdot \nabla_{\perp} N \\
& =\left(\nabla_{\perp} N \times \mathbf{b}\right) \cdot\left(\nabla_{\perp} \phi \cdot \nabla_{\perp} \nabla_{\perp} \phi\right) \\
& =\frac{1}{2}(\nabla N \times \mathbf{b}) \cdot\left(\nabla_{\perp} \nabla_{\perp} \phi^{2}\right)=\frac{1}{2}(\nabla N \times \mathbf{b}) \cdot \nabla_{\perp} \mathbf{v}_{E}^{2}
\end{aligned}
$$

Collecting all terms, we can write the equation for the evolution of potential vorticity:

$$
\partial_{t} \varpi=-\mathbf{v}_{E} \cdot \nabla \varpi+\nabla_{\|}\left(N v_{\|}\right)+\frac{1}{2}(\mathbf{b} \times \nabla N) \cdot \nabla_{\perp} \mathbf{v}_{E}^{2}-\nu_{i n} \varpi
$$

\section{Appendix C: Parameters and profiles for the benchmark case}

Parameters and profiles used for the simulation are presented in Fig. 8 ,

Common parameters for all 3 cases (drift wave, Kelvin-Helmholtz, interchange):

Helium plasma, once ionized $Z=1$

Radial interval $r_{a} \leq r \leq r_{b}, r_{a}=0.15 \mathrm{~m}, r_{b}=0.45 \mathrm{~m}$

$B_{0}=0.04 \mathrm{~T}, T_{e}=5 \mathrm{eV}, \nu_{i n}=0, L_{z}=17 \mathrm{~m}$

Density profile is a polynomial fit to the experimental profile $n_{i}(r)=n_{0} \sum_{i=0}^{5} c_{i} r^{i}$,

$\left\{c_{i}\right\}=\{-5.4638,124.624,-882.24,2863.636,-4436.36,2666.664\}, n_{0}=2.5 \times 10^{18} \mathrm{~m}^{-3}$.

Different parameters for each of the 3 cases:

Drift wave case: $n_{z}=0.5, \phi_{0}(r)=0$.

Kelvin-Helmholtz case: $n_{z}=0$,

$$
\begin{aligned}
& \phi_{0}(r)=\phi_{x}(F(x-1)+F(x+1)-2 F(x)), \\
& F(x)=\frac{1}{8}\left(\frac{2 w x}{\sqrt{\pi}} e^{-\frac{x^{2}}{w^{2}}}+\left(w^{2}+2 x^{2}\right) \operatorname{erf}\left(\frac{x}{w}\right)\right),
\end{aligned}
$$


$x=4\left(r-r_{a}\right) /\left(r_{b}-r_{a}\right)-2, w=0.8, \phi_{x}=50 \mathrm{~V}$.

Interchange case: $n_{z}=0, \phi_{0}(r)=\phi_{x}\left(\frac{r}{r_{b}}\right)^{2}, \phi_{x}=50 \mathrm{~V}$.

Boundary conditions: periodic in the azimuthal and axial directions; $\phi\left(r_{a}\right)=\phi\left(r_{b}\right)=0$ radially.

\section{REFERENCES}

${ }^{1}$ M. Greenwald, Phys. Plasmas 17, 058101 (2010).

${ }^{2}$ V. Naulin, T. Windisch, and O. Grulke, Physics of Plasmas 4, 012307 (2008).

${ }^{3}$ N. Kasuya, M. Yagi, M. Azumi, K. Itoh, and S.-I. Itoh, Journal of the Physical Society of Japan 76, 044501 (2007).

${ }^{4}$ C. Holland, G. R. Tynan, J. H. Yu, A. James, D. Nishijima, M. Shimada, and N. Taheri, Plasma Phys. Control. Fusion 49, A109 (2007).

${ }^{5}$ W. Gekelman, H. Pfister, Z. Lucky, J. Bamber, D. Leneman, and J. Maggs, Rev. Sci. Inst. 62, $2875(1991)$.

${ }^{6}$ X. Q. Xu and R. H. Cohen, Contrib. Plasma Phys. 36, 158 (1998).

${ }^{7}$ M. Umansky, X. Xu, B. Dudson, L. LoDestro, and J. Myra, Contrib. Plasma Phys. 180, $887(2009)$.

${ }^{8}$ J. R. Peñano, G. J. Morales, and J. E. Maggs, Phys. Plasmas 4, 555 (1997).

${ }^{9}$ W. Horton, J. C. Perez, T. Carter, and R. Bengtson, Physics of Plasmas 12, 022303 (2005).

${ }^{10}$ T. A. Carter and J. E. Maggs, Physics of Plasmas 16, 012304 (2009).

${ }^{11}$ E. M. Marshall, R. F. Ellis, and J. E. Walsh, Plasma Phys. Control. Fusion 28, 1461 (1986).

${ }^{12}$ T. D. Rognlien, J. Appl. Phys. 4, 3505 (1973).

${ }^{13}$ A. T. Burke, J. E. Maggs, and G. J. Morales, Phys. Rev. Lett. 84, 1451 (2000).

${ }^{14}$ J. E. Maggs and G. J. Morales, Phys. Plasmas 10, 2267 (2003).

${ }^{15}$ P. Popovich, M. Umansky, T. A. Carter, and B. Friedman, "Modeling of plasma turbulence and transport in the Large Plasma Device," arXiv:1005.2418, submitted to Phys. Plasmas.

${ }^{16}$ S. I. Braginskii, Transport processes in a plasma, in Reviews of Plasma Physics, edited by M. A. Leontovich, volume 1, pages 205-311, Consultants Bureau, New York, 1965.

${ }^{17}$ S. Vincena, W. Gekelman, and J. E. Maggs, Phys. Plasmas 8, 3884 (2001). 
${ }^{18}$ M. Ono and R. Kulsrud, Phys. Fluids 18, 1287 (1975).

${ }^{19}$ G. D. Byrne and A. C. Hindmarsh, International Journal of High Performance Computing Applications 13, 354 (1999).

${ }^{20}$ A. N. Simakov and P. J. Catto, Physics of Plasmas 10, pp. 4744 (2003).

${ }^{21}$ F. F. Chen, Introduction to Plasma Physics and Controlled Fusion, Plenum Press, New York, 1984.

${ }^{22}$ J. E. Maggs, T. A. Carter, and R. J. Taylor, Physics of Plasmas 14, 052507 (2007).

${ }^{23}$ G. Ganguli, Physics of Plasmas 4, 1544 (1997).

${ }^{24}$ M. Abramowitz and I. A. Stegun, Handbook of Mathematical Functions with Formulas, Graphs, and Mathematical Tables, Dover, New York, 1964.

${ }^{25}$ B. B. Kadomtsev, Plasma Turbulence, Academic Press, London, 1965.

${ }^{26}$ J. Wesson, Tokamaks, Oxford University Press, Oxford, 2004. 